\title{
Alkyl C-O Ring Cleavage of Bicyclic $\beta$-Lactones with Normant Reagents: Synthesis of a Merck IND Intermediate
}

\author{
Wei Zhang, Andrea Matla and Daniel Romo* \\ Department of Chemistry, P. O. Box 30012, Texas A\&M University, College Station, TX-77842-3012
}

Supporting Information Available: Detailed procedures and characterization data (Including ${ }^{1} \mathrm{H}$ and ${ }^{13} \mathrm{C}$ NMR spectra) for compound 2a-d, 3, 4, 6, 8, 10a, 11b-c, Merck IND intermediate 12, and 13b-c. This material is available free of charge via the Internet at http://pubs.acs.org.

\begin{tabular}{|c|c|c|c|}
\hline General Experimental & $\mathrm{S} 2$ & ${ }^{13} \mathrm{C}$ NMR 2c & S19 \\
\hline Procedure A & S3 & ${ }^{1} \mathrm{H}$ NMR 2d & $\mathrm{S} 20$ \\
\hline $2 \mathbf{a}$ & S3 & 13 C NMR 2d & $\mathrm{S} 21$ \\
\hline $2 \mathbf{b}$ & S3 & ${ }^{1} \mathrm{H}$ NMR 3 & $\mathrm{~S} 22$ \\
\hline $2 \mathrm{c}$ & S4 & ${ }^{13} \mathrm{C}$ NMR 3 & $\mathrm{~S} 23$ \\
\hline 2d & S4 & ${ }^{1} \mathrm{H}$ NMR 4 & $\mathrm{~S} 24$ \\
\hline 3 & S4 & ${ }^{13} \mathrm{C}$ NMR 4 & S25 \\
\hline 4 & S5 & ${ }^{1} \mathrm{H}$ NMR 6a & S26 \\
\hline Procedure B & S6 & 13 C NMR 6a & S27 \\
\hline Procedure C & S7 & ${ }^{1} \mathrm{H}$ NMR 8a & $\mathrm{S} 28$ \\
\hline $6 \mathbf{a}$ & S8 & ${ }^{13} \mathrm{C}$ NMR 8a & $\mathrm{S} 29$ \\
\hline 8 & S8 & ${ }^{1} \mathrm{H}$ NMR 10a & $\mathrm{S} 30$ \\
\hline 10a & S8 & ${ }^{13}$ C NMR 10a & S31 \\
\hline 11b & S9 & ${ }^{1} \mathrm{H}$ NMR 11b & $\mathrm{S} 32$ \\
\hline 11c & S9 & ${ }^{13} \mathrm{C}$ NMR 11b & S33 \\
\hline$m$-F-PhMgCl & $\mathrm{S} 10$ & ${ }^{1} \mathrm{H}$ NMR 11c & S34 \\
\hline 13b & S10 & ${ }^{13} \mathrm{C}$ NMR 11c & S35 \\
\hline$m$-OMe-PhMgCl & S11 & ${ }^{1} \mathrm{H}$ NMR 12 & S36 \\
\hline 13c & S11 & ${ }^{13} \mathrm{C}$ NMR 12 & S37 \\
\hline 12 & $\mathrm{~S} 12$ & ${ }^{1} \mathrm{H}$ NMR 13b & S38 \\
\hline${ }^{1} \mathrm{H}$ NMR 2a & S14 & ${ }^{13} \mathrm{C}$ NMR 13b & S39 \\
\hline${ }^{13} \mathrm{C}$ NMR 2a & S15 & ${ }^{1} \mathrm{H}$ NMR 13c & $\mathrm{S} 40$ \\
\hline${ }^{1} \mathrm{H}$ NMR 2b & S16 & ${ }^{13} \mathrm{C}$ NMR 13c & S41 \\
\hline 13 C NMR 2b & S17 & & \\
\hline${ }^{1} \mathrm{H}$ NMR 2c & S18 & & \\
\hline
\end{tabular}




\section{General Procedure}

All reactions were carried out under nitrogen atmosphere in flame-dried glassware. Dichloromethane, methanol, diethyl ethere were purified by passage through activated molecular sieves or alumina (solvent system). tetrahydrofuran was freshly distilled over sodium and benzophenone. $\mathrm{CuBr} \bullet \mathrm{DMS}$ and $\mathrm{CuCl}$ as well as commercially available Grignard reagents were purchased from Aldrich. TMSCl was distilled from calcium hydride prior to use. All other commercial reagents were used as received. ${ }^{1} \mathrm{H}$ NMR chemical shifts are reported as $\delta$ values in ppm relative to $\mathrm{CDCl}_{3}(7.29 \mathrm{ppm}$,$) ,$ coupling constants $(J)$ are reported in Hertz $(\mathrm{Hz})$, and multipilicity follows convention. Unless indicated otherwise, deuterochloroform $\left(\mathrm{CDCl}_{3}\right)$ served as an internal standard (77.0 ppm) for all ${ }^{13} \mathrm{C}$ spectra. Flash column chromatography was performed using $60 \AA$ Silica Gel (Baker, 230-400 mesh or Silacycle, 230-400 mesh) as a stationary phase. Mass spectra were obtained at the center for Chemical Characterization and Analysis (Texas A\&M University). Thin layer chromatography (TLC) was performed using glass-backed silica gel 60 $0_{\mathrm{F} 254}($ Merck, $250 \mu \mathrm{m}$ thickness). Bicyclic $\beta$-lactones (+)-1 and (+)-9 were prepared as previously described. ${ }^{1}$

\footnotetext{
${ }^{1}$ (a) Cortez, G. S.; Tennyson, R. L.; Romo, D. J. Am. Chem. Soc. 2001, 123, 7945. (b) Oh, S. H.; Cortez, G. S.; Romo, D. J. Org. Chem. 2005, 70, 2835.
} 
Representative procedure A for $\beta$-lactone cleavage with alkyl cuprates (Table 1, entry 1) as described for (+)-(7S,8S)-8-methyl-1,4-dioxaspiro[4.4]nonane-7carboxylic acid 2a:

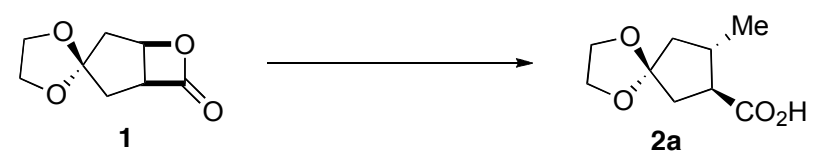

To a suspension of $\mathrm{CuBr} \cdot \mathrm{DMS}(400 \mathrm{mg}, 1.95 \mathrm{mmol})$ in THF $(8.0 \mathrm{~mL})$ at $23^{\circ} \mathrm{C}$ was added $\mathrm{Me}_{2} \mathrm{~S}$ ( $\left.274 \mathrm{mg}, 0.33 \mathrm{~mL}, 4.42 \mathrm{mmol}\right)$. After this mixture was cooled to $-42^{\circ} \mathrm{C}$, a solution of $\mathrm{MeMgBr}$ in THF $(1.74 \mathrm{~mL}, 3.89 \mathrm{mmol}, 2.24 \mathrm{M}$ in THF) was added dropwise. After $30 \mathrm{~min}$ at $-42^{\circ} \mathrm{C}$ and $30 \mathrm{~min}$ at $-30^{\circ} \mathrm{C}$, it was cooled back to $-42^{\circ} \mathrm{C}$. A solution of $\mathbf{1}(110$ $\mathrm{mg}, 0.65 \mathrm{mmol})$ in THF $(1.5 \mathrm{~mL})$ was added dropwise via cannula. After $10 \mathrm{~min}$, TMSCl (108 mg, $0.12 \mathrm{~mL}, 0.98 \mathrm{mmol}$ ) was added dropwise and the reaction was slowly warmed to $-5^{\circ} \mathrm{C}$ over $2 \mathrm{~h}$ and quenched with $1 \mathrm{~N} \mathrm{HCl}(5.0 \mathrm{~mL})$ and sat. aq. $\mathrm{NH}_{4} \mathrm{Cl}(10.0$ $\mathrm{mL})$. The mixture was extracted with $\mathrm{Et}_{2} \mathrm{O}(3 \mathrm{X} 20 \mathrm{~mL})$ and the combined organics were washed with sat. aq. $\mathrm{NH}_{4} \mathrm{Cl}(1 \mathrm{X} 20 \mathrm{~mL})$ and aq. brine solution $(2 \mathrm{X} 20 \mathrm{~mL})$. The dried $\left(\mathrm{MgSO}_{4}\right)$ extract was concentrated in vacuo and purified by flash chromatography, eluting with EtOAc / hexanes / acetic acid (20:80:1), to give 2a (74 mg, $0.40 \mathrm{mmol}, 62 \%$ ) as a colorless oil: $[\alpha]_{\mathrm{D}}{ }^{23}+13.3\left(c 1.80, \mathrm{CHCl}_{3}\right.$ ); IR (thin film) 3482 (br), $3203(\mathrm{br}), 2965$, 1703, 1432, 1323, $1159 \mathrm{~cm}^{-1}$; ${ }^{1} \mathrm{H}$ NMR $\left(500 \mathrm{MHz}, \mathrm{CDCl}_{3}\right) \delta$ 3.87-3.97 (m, 4H), 2.50 (dd, $J=9.5,18.5 \mathrm{~Hz}, 1 \mathrm{H}), 2.32-2.39$ (m, 1H), 2.13-2.24 (m, 3H), 1.55 (dd, $J=10.5,13.5 \mathrm{~Hz}$, $1 \mathrm{H}), 1.15(\mathrm{~d}, J=6.5 \mathrm{~Hz}, 3 \mathrm{H}) ;{ }^{13} \mathrm{C}$ NMR $\left(125 \mathrm{MHz}, \mathrm{CDCl}_{3}\right) \delta 180.8,116.3,64.6(2 \mathrm{C})$, 49.6, 44.7, 40.4, 36.6, 19.7; LRMS (ESI+) calcd. for $\mathrm{C}_{9} \mathrm{H}_{14} \mathrm{O}_{4}(\mathrm{M}+\mathrm{H})$ 187, found 187 .

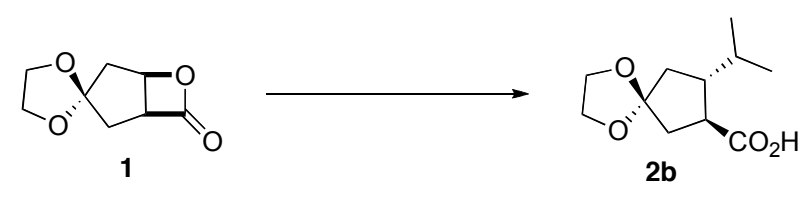

(+)-(7S,8R)-8-isopropyl-1,4-dioxaspiro[4.4]nonane-7-carboxylic acid 2b was prepared according to the representative procedure A using CuBr$• D M S ~(265 \mathrm{mg}, 1.29 \mathrm{mmol})$, $\mathrm{Me}_{2} \mathrm{~S}$ (181 mg, $\left.0.21 \mathrm{~mL}, 2.9 \mathrm{mmol}\right), i-\mathrm{PrMgCl}(1.29 \mathrm{~mL}, 2.58 \mathrm{mmol}, 2.0 \mathrm{M}$ in THF), bicyclic $\beta$-lactone 1 (73 mg, $0.43 \mathrm{mmol})$ and TMSCl $(70 \mathrm{mg}, 81 \mu \mathrm{L}, 0.65 \mathrm{mmol})$. 
Purification by flash chromatography eluting with EtOAc / hexanes / acetic acid (20:80:1) gave $2 \mathbf{b}(51 \mathrm{mg}, 0.24 \mathrm{mmol}, 56 \%)$ as a colorless oil: $[\alpha]_{\mathrm{D}}{ }^{23}+10.5(c 1.90$, $\mathrm{CHCl}_{3}$ ); IR (thin film) 3478 (br), 3200 (br), 2960, 1706, 1319, $1119 \mathrm{~cm}^{-1}$; ${ }^{1} \mathrm{H}$ NMR (500 $\left.\mathrm{MHz}, \mathrm{CDCl}_{3}\right)$ d 3.87-3.98 (m, 4H), 2.64-2.71 (m, 1H), 2.22-2.29 (m, 1H), 2.19 (dd, $J=$ 9.0, $13.5 \mathrm{~Hz}, 1 \mathrm{H}), 2.11(\mathrm{dd}, J=9.0,13.5 \mathrm{~Hz}, 1 \mathrm{H}), 2.04(\mathrm{dd}, J=8.0,13.0 \mathrm{~Hz}, 1 \mathrm{H}), 1.66-$ $1.76(\mathrm{~m}, 1 \mathrm{H}), 1.64(\mathrm{dd}, J=10.5,13.0 \mathrm{~Hz}, 1 \mathrm{H}), 0.92(\mathrm{~d}, J=6.5 \mathrm{~Hz}, 3 \mathrm{H}), 0.89(\mathrm{~d}, J=7.0$ $\mathrm{Hz}, 3 \mathrm{H}) ;{ }^{13} \mathrm{C}$ NMR $\left(125 \mathrm{MHz}, \mathrm{CDCl}_{3}\right) \delta 181.6,116.2,64.67,64.65,47.3,40.6,40.0$, 31.7, 21.2, 19.5 ; LRMS (ESI+) calcd. for $\mathrm{C}_{11} \mathrm{H}_{17} \mathrm{O}_{4}(\mathrm{M}-\mathrm{H})$ 213, found 213.

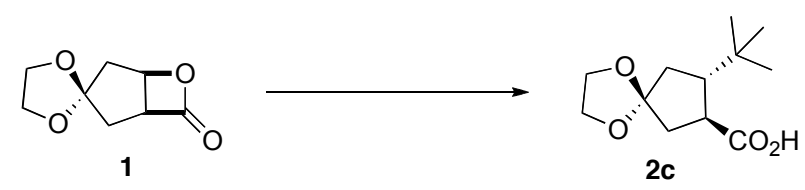

(+)-(7S,8S)-8-tert-butyl-1,4-dioxaspiro[4.4]nonane-7-carboxylic acid 2c was prepared

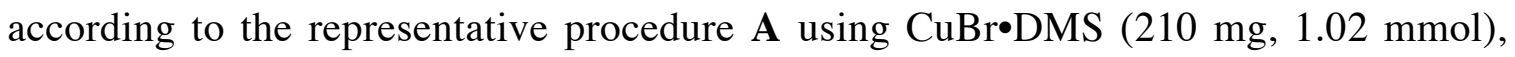
$\mathrm{Me}_{2} \mathrm{~S}(142 \mathrm{mg}, 0.17 \mathrm{~mL}, 2.3 \mathrm{mmol}), t-\mathrm{BuMgBr}(1.0 \mathrm{~mL}, 2.0 \mathrm{mmol}, 2.0 \mathrm{M}$ in THF), bicyclic $\beta$-lactone $1(58 \mathrm{mg}, 0.34 \mathrm{mmol})$ and $\mathrm{TMSCl}(56 \mathrm{mg}, 64 \mu \mathrm{L}, 0.51 \mathrm{mmol})$. Purification by flash chromatography, eluting with EtOAc / hexanes / acetic acid (20:80:1) gave 2 c (19 mg, $0.09 \mathrm{mmol}, 25 \%)$ as a colorless oil: $[\alpha]_{\mathrm{D}}{ }^{23}+6.9$ (c 1.16, $\mathrm{CHCl}_{3}$ ); IR (thin film) 3468 (br), 3199 (br), 2960, 1690, 1466, 1324, $1019 \mathrm{~cm}^{-1} ;{ }^{1} \mathrm{H}$ NMR (500 MHz, $\left.\mathrm{CDCl}_{3}\right) \delta 3.87-3.98(\mathrm{~m}, 4 \mathrm{H}), 2.71-2.79(\mathrm{~m}, 1 \mathrm{H}), 2.43$ (dd, $J=9.0,19.0 \mathrm{~Hz}$, 1H), 2.17 (dd, $J=10.5,14.0 \mathrm{~Hz}, 1 \mathrm{H}), 2.09$ (dd, $J=7.5,14.0 \mathrm{~Hz}, 1 \mathrm{H}), 1.97$ (dd, $J=8.5$, $12.5 \mathrm{~Hz}, 1 \mathrm{H}), 1.72$ (dd, $J=10.5,13.0 \mathrm{~Hz}, 1 \mathrm{H}), 0.90$ (s, 9H); ${ }^{13} \mathrm{C}$ NMR $(125 \mathrm{MHz}$, $\left.\mathrm{CDCl}_{3}\right) \delta 181.4,116.3,64.9,64.7,50.2,40.8,37.6,32.9,29.6,27.5$; LRMS (ESI+) Calcd. for $\mathrm{C}_{12} \mathrm{H}_{19} \mathrm{O}_{4}(\mathrm{M}-\mathrm{H})$ : 227. Found: 227.

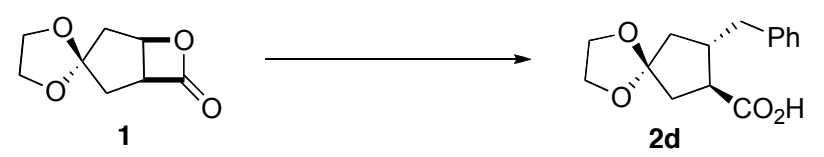

(+)-(7S,8S)-8-benzyl-1,4-dioxaspiro[4.4]nonane-7-carboxylic acid 2d was prepared according to the representative procedure A using CuBr$• D M S ~(154 \mathrm{mg}, 0.75 \mathrm{mmol}$ ), $\mathrm{Me}_{2} \mathrm{~S}$ (106 mg, $\left.0.13 \mathrm{~mL}, 1.7 \mathrm{mmol}\right), \mathrm{BnMgBr}(0.75 \mathrm{~mL}, 1.5 \mathrm{mmol}, 2.0 \mathrm{M}$ in THF), 
bicyclic $\beta$-lactone 1 (42 $\mathrm{mg}, 0.25 \mathrm{mmol})$ and $\mathrm{TMSCl}(42 \mathrm{mg}, 50 \mu \mathrm{L}, 0.38 \mathrm{mmol})$. Purification by flash chromatography eluting with EtOAc / hexanes / acetic acid (20:80:1) gave 2d (51 mg, $0.19 \mathrm{mmol}, 78 \%)$ as a white solide: $[\alpha]_{\mathrm{D}}{ }^{23}+24.6(c) 1.22$, $\mathrm{CHCl}_{3}$ ); IR (thin film) 3469 (br), 3234 (br), 2953, 1707, 1335, $1124 \mathrm{~cm}^{-1} ;{ }^{1} \mathrm{H}$ NMR (500 $\left.\mathrm{MHz}, \mathrm{CDCl}_{3}\right) \delta$ 7.26-7.36 (m, 2H), 7.18-7.24 (m, 3H), 3.83-3.95 (m, 4H), 3.03 (d, J = 9.5 $\mathrm{Hz}, 1 \mathrm{H}), 2.60-2.70$ (m, 3H), 2.19-2.29 (m, 2H), 2.01 (dd, $J=6.5,13.5 \mathrm{~Hz}, 1 \mathrm{H}), 1.63$ (dd, $J=9.0,13.5 \mathrm{~Hz}, 1 \mathrm{H}) ;{ }^{13} \mathrm{C}$ NMR $\left(125 \mathrm{MHz}, \mathrm{CDCl}_{3}\right) \delta 181.0,140.1,129.3,128.6,126.4$, 116.1, 64.64, 64.61, 47.5, 42.8, 41.8, 40.8, 40.1; LRMS (ESI+) Calcd. for $\mathrm{C}_{15} \mathrm{H}_{17} \mathrm{O}_{4}$ (MH): 261. Found: 261.

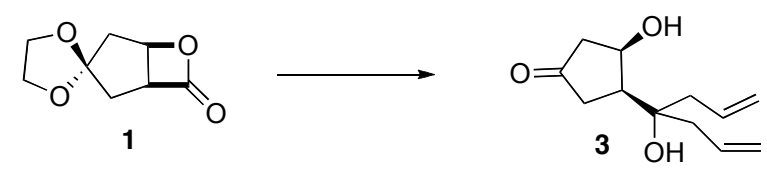

(+)-(3R,4S)-3-hydroxy-4-(4-hydroxyhepta-1,6-dien-4-yl)cyclopentanone 3 was prepared

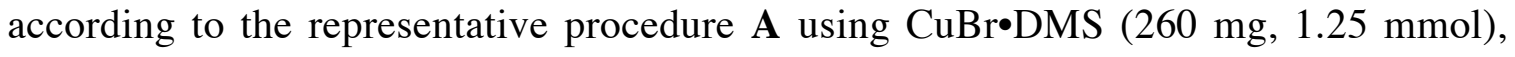
$\mathrm{Me}_{2} \mathrm{~S}$ (177 mg, $0.21 \mathrm{~mL}, 2.9 \mathrm{mmol}$ ), allyl $\mathrm{MgBr}$ (1.25 mL, $2.5 \mathrm{mmol}, 2.0 \mathrm{M}$ in THF), bicyclic $\beta$-lactone $1(72 \mathrm{mg}, 0.42 \mathrm{mmol})$ and $\mathrm{TMSCl}(68 \mathrm{mg}, 80 \mu \mathrm{L}, 0.63 \mathrm{mmol})$. Purification by flash chromatography eluting with EtOAc / hexanes (20:80) gave 3 (73 $\mathrm{mg}, 0.35 \mathrm{mmol}, 82 \%$ ) as a colorless oil: $[\alpha]_{\mathrm{D}}^{23}+97.2\left(c 1.42, \mathrm{CHCl}_{3}\right)$; IR (thin film) 3367 (br), 2925, 1738, 1431, $1158 \mathrm{~cm}^{-1} ;{ }^{1} \mathrm{H}$ NMR $\left(500 \mathrm{MHz}, \mathrm{CDCl}_{3}\right) \delta 5.88-5.96(\mathrm{~m}, 1 \mathrm{H})$, 5.74-5.82 (m, 1H), 5.13-5.25 (m, 4H), 4.79-4.83 (m, 1H), $4.23(\mathrm{~s}, 1 \mathrm{H}), 3.34(\mathrm{~s}, 1 \mathrm{H}), 2.66-$ $2.76(\mathrm{~m}, 1 \mathrm{H}), 2.60(\mathrm{ddd}, J=0.5,6.5,13.5 \mathrm{~Hz}, 1 \mathrm{H}), 2.50(\mathrm{dd}, J=8.0,14.0 \mathrm{~Hz}, 1 \mathrm{H}), 2.15-$ $2.42(\mathrm{~m}, 6 \mathrm{H}) ;{ }^{13} \mathrm{C} \mathrm{NMR}\left(125 \mathrm{MHz}, \mathrm{CDCl}_{3}\right) \delta$ 217.5, 133.2, 132.6, 119.6 (2C), 75.5, 71.1, 49.8, 46.6, 43.1, 42.0, 35.4; LRMS (ESI+) Calcd. for $\mathrm{C}_{12} \mathrm{H}_{19} \mathrm{O}_{3}(\mathrm{M}+\mathrm{H})$ : 211. Found: 211.

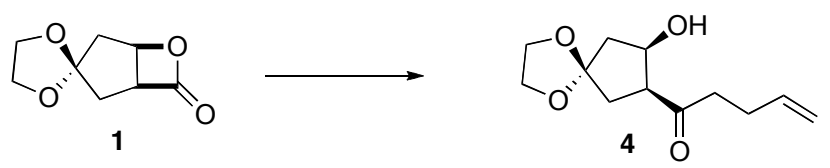

(-)-1-((7S,8R)-8-hydroxy-1,4-dioxaspiro[4-4]nonan-7-yl)pent-4-en-1-one 4 was prepared according to the representative procedure $\mathbf{A}$ using CuBr$\cdot D M S$ (185 $\mathrm{mg}, 0.9 \mathrm{mmol}$ ), $\mathrm{Me}_{2} \mathrm{~S}$ (127 mg, $0.15 \mathrm{~mL}, 2.0 \mathrm{mmol}$ ), vinyl $\mathrm{MgBr}(0.9 \mathrm{~mL}, 1.8 \mathrm{mmol}, 2.0 \mathrm{M}$ in THF), bicyclic $\beta$-lactone 1 (51 mg, $0.3 \mathrm{mmol}$ ) and TMSCl (49 mg, $57 \mu \mathrm{L}, 0.45 \mathrm{mmol}$ ). Purification by 
flash chromatography eluting with EtOAc / hexanes (30:70) gave 4 (61 mg, $0.27 \mathrm{mmol}$, 90\%) as a colorless oil: $[\alpha]_{\mathrm{D}}^{23}-11.4\left(c 0.88, \mathrm{CHCl}_{3}\right)$; IR (thin film) $3479(\mathrm{br}), 2952$, 1712, 1331, 1116, $1017 \mathrm{~cm}^{-1} ;{ }^{1} \mathrm{H}$ NMR $\left(500 \mathrm{MHz}, \mathrm{CDCl}_{3}\right) \delta$ 5.76-5.84 (m, 1H), 4.95$5.04(\mathrm{~m}, 2 \mathrm{H}), 4.54-4.58(\mathrm{~m}, 1 \mathrm{H}), 3.86-3.98(\mathrm{~m}, 4 \mathrm{H}), 3.09$ (dt, $J=5.0,10.0 \mathrm{~Hz}, 2 \mathrm{H}), 2.67$ (dt, $J=7.5,17.0 \mathrm{~Hz}, 1 \mathrm{H}), 2.53$ (dt, $J=7.5,17.5 \mathrm{~Hz}, 1 \mathrm{H}), 2.46$ (dd, $J=10.0,14.0 \mathrm{~Hz}$, $1 \mathrm{H}$ ), 2.33 (dd, $J=6.5,14.0 \mathrm{~Hz}, 2 \mathrm{H}$ ), 2.10 (dd, $J=5.0,14.5 \mathrm{~Hz}, 1 \mathrm{H}$ ), 1.99 (dd, $J=9.0$, $14.0 \mathrm{~Hz}, 1 \mathrm{H}) ;{ }^{13} \mathrm{C} \mathrm{NMR}\left(125 \mathrm{MHz}, \mathrm{CDCl}_{3}\right) \delta$ 209.9, 137.3, 116.5, 115.5, 72.3, 64.8, 64.6, 55.2, 45.5, 41.6, 35.5, 27.6; LRMS (ESI+) Calcd. for $\mathrm{LiC}_{12} \mathrm{H}_{18} \mathrm{O}_{4}(\mathrm{M}+\mathrm{Li})$ : 233. Found: 233.

Representative procedure $B$ for $\beta$-lactone opening with aryl cuprates derived from chloro Grignard reagents and $\mathrm{CuBr}^{\circ} \mathrm{Me}_{2} \mathrm{~S}$ and esterification (Table 2, entry 7) as described for (+)-(7S,8S)-methyl 8-phenyl-1,4-dioxaspiro[4.4]nonane-7-carboxylate 6a and (+)-(7R,8S)-methyl 8-bromo-1,4-dioxaspiro[4.4]nonane-7-carboxylate 8a

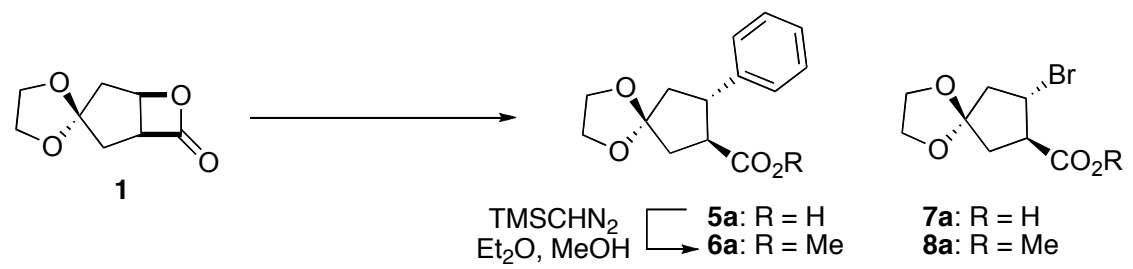

To a suspension of $\mathrm{CuBr} \bullet \mathrm{DMS}$ ( $310 \mathrm{mg}, 1.5 \mathrm{mmol})$ in THF $(5.0 \mathrm{~mL})$ at $23^{\circ} \mathrm{C}$ was added $\mathrm{Me}_{2} \mathrm{~S}^{2}$ (268 mg, $0.32 \mathrm{~mL}, 4.3 \mathrm{mmol}$ ). After this mixture was cooled to $-42^{\circ} \mathrm{C}$, a solution of $\mathrm{PhMgCl}$ in THF (1.5 mL, $3.0 \mathrm{mmol}, 2.0 \mathrm{M}$ in THF) was added dropwise. After $30 \mathrm{~min}$ at $-42^{\circ} \mathrm{C}$ and $30 \mathrm{~min}$ at $-30^{\circ} \mathrm{C}$, it was cooled back to $-42^{\circ} \mathrm{C}$. A solution of 1 (45 mg, $0.27 \mathrm{mmol})$ in THF (1.2 mL) was added dropwise. After $10 \mathrm{~min}$, TMSCl (57 $\mathrm{mg}, 68 \mu \mathrm{L}, 0.53 \mathrm{mmol}$ ) was added dropwise and the reaction was slowly warmed up to $10^{\circ} \mathrm{C}$ within $4 \mathrm{~h}$. After $4 \mathrm{~h}$ at $10^{\circ} \mathrm{C}$, it was quenched with $1 \mathrm{~N} \mathrm{HCl}(5.0 \mathrm{~mL})$ and sat. aq. $\mathrm{NH}_{4} \mathrm{Cl}(10.0 \mathrm{~mL})$. The mixture was extracted with $\mathrm{Et}_{2} \mathrm{O}(3 \mathrm{X} 40 \mathrm{~mL})$ and the combined organics were washed with sat. aq. $\mathrm{NH}_{4} \mathrm{Cl}(1 \mathrm{X} 20 \mathrm{~mL})$ and aq. brine solution $(2 \mathrm{X} 20$ $\mathrm{mL})$. The dried $\left(\mathrm{MgSO}_{4}\right)$ extract was concentrated in vacuo and purified by

\footnotetext{
${ }^{2}$ A systematic study of the equivalents of $\mathrm{Me}_{2} \mathrm{~S}$ employed showed that use of excess $\mathrm{Me}_{2} \mathrm{~S}$ led to a homogeneous cuprate solution. It was determined that 16 equiv $\mathrm{Me}_{2} \mathrm{~S}$ (relative to substrate) was an optimal quantity for achieving homogeniety.
} 
chromatography over a short plug of silica gel, eluting with EtOAc / hexanes / acetic acid (20:80:1), to give a mixture of aryl adduct $\mathbf{5 a}$ and bromo acid $\mathbf{7 a}{ }^{3}$ (The end of the procedure $\mathrm{B}$ )

To this mixture in a solution of $\mathrm{Et}_{2} \mathrm{O} / \mathrm{MeOH}(3.0 \mathrm{~mL} / 1.0 \mathrm{~mL})$ at $23^{\circ} \mathrm{C}$ was added a solution of $\mathrm{TMSCHN}_{2}(0.23 \mathrm{~mL}, 0.46 \mathrm{mmol}, 2.0 \mathrm{M}$ in THF). After $5 \mathrm{~min}$, the solvent was removed in vacuo and purified by chromatography over a short plug of silica gel, eluting with $1 \%$ EtOAc $/ \mathrm{CH}_{2} \mathrm{Cl}_{2}$, to give ester 6a (50 mg, $0.19 \mathrm{mmol}, 72 \%$ ) as a colorless oil and bromoester $8 \mathbf{a}$ (13 $\mathrm{mg}, 0.05 \mathrm{mmol}, 18 \%)$ as a colorless oil.

\section{Representative procedure $C$ for $\beta$-lactone opening with aryl cuprates derived from} Chloro Grignard reagents and $\mathrm{CuCl}$ (Table 2, entry 8) as described for (+)-(7S,8S)methyl 8-phenyl-1,4-dioxaspiro[4.4]nonane-7-carboxylate 6a and (+)-(7R,8S)-methyl

\section{8-bromo-1,4-dioxaspiro[4.4]nonane-7-carboxylate 8a}

To a suspension of $\mathrm{CuCl}(150 \mathrm{mg}, 1.5 \mathrm{mmol})$ in $\mathrm{THF}(5.0 \mathrm{~mL})$ at $23{ }^{\circ} \mathrm{C}$ was added $\mathrm{Me}_{2} \mathrm{~S}\left(300 \mathrm{mg}, 0.35 \mathrm{~mL}, 4.3 \mathrm{mmol}\right.$ ). After this mixture was cooled to $-42^{\circ} \mathrm{C}$, a solution of $\mathrm{PhMgCl}$ in THF (1.5 mL, $3.0 \mathrm{mmol}, 2.0 \mathrm{M}$ in THF) was added dropwise. After $30 \mathrm{~min}$ at $-42^{\circ} \mathrm{C}$ and $1 \mathrm{~h}$ at $-20^{\circ} \mathrm{C}$, it was cooled back to $-42^{\circ} \mathrm{C}$. A solution of $\mathbf{1}$ $(51 \mathrm{mg}, 0.30 \mathrm{mmol})$ in THF $(1.2 \mathrm{~mL})$ was added dropwise. After $10 \mathrm{~min}$, TMSCl $(65$ $\mathrm{mg}, 76 \mu \mathrm{L}, 0.60 \mathrm{mmol}$ ) was added dropwise and the reaction was slowly warmed up to $10^{\circ} \mathrm{C}$ within $4 \mathrm{~h}$. After $4 \mathrm{~h}$ at $10^{\circ} \mathrm{C}$, it was quenched with $1 \mathrm{~N} \mathrm{HCl}(5.0 \mathrm{~mL})$ and sat. aq. $\mathrm{NH}_{4} \mathrm{Cl}(10.0 \mathrm{~mL})$. The mixture was extracted with $\mathrm{Et}_{2} \mathrm{O}(3 \mathrm{X} 40 \mathrm{~mL})$ and the combined organics were washed with sat. aq. $\mathrm{NH}_{4} \mathrm{Cl}(1 \mathrm{X} 20 \mathrm{~mL})$ and aq. brine solution $(2 \mathrm{X} 20$ $\mathrm{mL}$ ). The dried $\left(\mathrm{MgSO}_{4}\right)$ extract was concentrated in vacuo. (The end of the procedure C)

To this crude mixture in a solution of $\mathrm{Et}_{2} \mathrm{O} / \mathrm{MeOH}(3.0 \mathrm{~mL} / 1.0 \mathrm{~mL})$ at $23^{\circ} \mathrm{C}$ was added a solution of $\mathrm{TMSCHN}_{2}(0.23 \mathrm{~mL}, 0.46 \mathrm{mmol}, 2.0 \mathrm{M}$ in THF $)$. After $5 \mathrm{~min}$, the solvent was removed in vacuo and purified by chromatography over a short plug of silica

${ }^{3}$ (7R,8S)-8-bromo-1,4-dioxaspiro[4.4]nonane-7-carboxylic acid $\mathbf{7}$ is somewhat unstable at ambient temperature once concentrated and would likely need to be carried directly to a subsequent reaction. 
gel, eluting with $1 \%$ EtOAc $/ \mathrm{CH}_{2} \mathrm{Cl}_{2}$, to give 6 (48 $\mathrm{mg}, 0.18 \mathrm{mmol}, 61 \%$ ) as a colorless oil.

(+)-(7S,8S)-methyl 8-phenyl-1,4-dioxaspiro[4.4]nonane-7-carboxylate 6a: $[\alpha]_{\mathrm{D}}{ }^{23}$ +38.2 (c 2.2, $\mathrm{CHCl}_{3}$ ); IR (thin film) 2956, 2889, 1735, 1435, 1330, $1161 \mathrm{~cm}^{-1} ;{ }^{1} \mathrm{H}$ NMR $\left(500 \mathrm{MHz}, \mathrm{CDCl}_{3}\right) \delta 7.20-7.33(\mathrm{~m}, 5 \mathrm{H}), 3.90-4.01(\mathrm{~m}, 4 \mathrm{H}), 3.60$ (s, 3H), $3.53(\mathrm{dt}, J=$ $8.5,10.5 \mathrm{~Hz}, 1 \mathrm{H}), 3.03(\mathrm{dt}, J=8.5,10.5 \mathrm{~Hz}, 1 \mathrm{H}), 2.40(\mathrm{dd}, J=8.5,13.0 \mathrm{~Hz}, 1 \mathrm{H}), 2.36$ (dd, $J=8.5,13.5 \mathrm{~Hz}, 1 \mathrm{H}), 2.23$ (ddd, $J=1.0,10.5,13.5 \mathrm{~Hz}, 1 \mathrm{H}$ ), 2.08 (ddd, $J=1.0,11.0$, $14.0 \mathrm{~Hz}, 1 \mathrm{H}) ;{ }^{13} \mathrm{C}$ NMR $\left(125 \mathrm{MHz}, \mathrm{CDCl}_{3}\right) \delta 174.8,142.8,128.8,127.5,126.9,115.9$, 64.8, 64.7, 52.0, 50.2, 46.7, 44.6, 41.0; LRMS (ESI+) Calcd. for $\mathrm{LiC}_{15} \mathrm{H}_{18} \mathrm{O}_{4}(\mathrm{M}+\mathrm{Li}): 269$. Found: 269.

(+)-(7R,8S)-methyl 8-bromo-1,4-dioxaspiro[4.4]nonane-7-carboxylate 8: $[\alpha]_{\mathrm{D}}{ }^{23}$ +48.4 (c 0.95, $\mathrm{CHCl}_{3}$ ); IR (thin film) 2958, 2892, 1740, 1434, 1327, 1168, $1017 \mathrm{~cm}^{-1} ;{ }^{1} \mathrm{H}$ NMR (500 MHz, $\left.\mathrm{CDCl}_{3}\right) \delta 4.48(\mathrm{dd}, J=8.0,17.0 \mathrm{~Hz}, 1 \mathrm{H}), 3.83-3.98(\mathrm{~m}, 4 \mathrm{H}), 3.61$ (s, $3 \mathrm{H}), 3.23(\mathrm{dd}, J=9.0,18.0 \mathrm{~Hz}, 1 \mathrm{H}), 2.62(\mathrm{dd}, J=8.0,14.5 \mathrm{~Hz}, 1 \mathrm{H}), 2.36(\mathrm{dd}, J=9.0$, $14.0 \mathrm{~Hz}, 1 \mathrm{H}$ ), 2.29 (ddd, $J=1.5,9.5,11.5 \mathrm{~Hz}, 1 \mathrm{H}), 2.10$ (ddd, $J=1.0,9.5,13.5 \mathrm{~Hz}, 1 \mathrm{H}$ ); ${ }^{13} \mathrm{C}$ NMR $\left(125 \mathrm{MHz}, \mathrm{CDCl}_{3}\right) \delta$ 173.0, 114.8, 64.9, 64.8, 52.7, 52.6, 47.4, 45.1, 40.4; LRMS (CI+) Calcd. for $\mathrm{C}_{9} \mathrm{H}_{14} \mathrm{BrO}_{4}(\mathrm{M}+\mathrm{H}): 265$. Found: 265 .

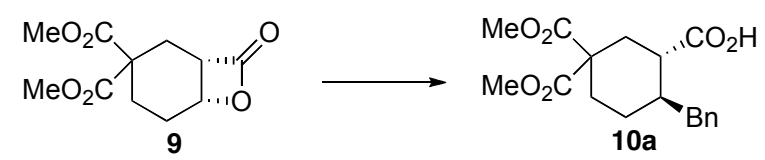

(+)-(1S,2R)-2-benzyl-5,5-bis(methoxycarbonyl)cyclohexanecarboxylic acid 10a was prepared according to the procedure $\mathbf{A}$ from $\mathrm{CuBr} \bullet \mathrm{DMS}$ (206 $\mathrm{mg}, 1.0 \mathrm{mmol}), \mathrm{Me}_{2} \mathrm{~S}$ (164 mg, $0.19 \mathrm{~mL}, 2.64 \mathrm{mmol}), \mathrm{BnMgCl}(1.0 \mathrm{~mL}, 2.0 \mathrm{mmol}, 2.0 \mathrm{M}$ in THF), bicyclic $\beta$ lactone 9 ( $80 \mathrm{mg}, 0.33 \mathrm{mmol})$ and TMSCl ( $54 \mathrm{mg}, 63 \mu \mathrm{L}, 0.5 \mathrm{mmol})$. Purified by flash chromatography, eluting with EtOAc / hexanes / acetic acid (20:80:1), to give 10a (84 $\mathrm{mg}, 0.25 \mathrm{mmol}, 76 \%)$ as a colorless oil: $[\alpha]_{\mathrm{D}}^{23}+11.0\left(c 1.00, \mathrm{CHCl}_{3}\right)$; IR (thin film) 3304 (br), 3031 (br), 2954, 1738, 1450, 1237, $1072 \mathrm{~cm}^{-1}$; ${ }^{1} \mathrm{H}$ NMR (500 MHz, $\left.\mathrm{CDCl}_{3}\right) \delta$ 7.28 (t, $J=7.5 \mathrm{~Hz}, 2 \mathrm{H}), 7.20$ (t, $J=7.5 \mathrm{~Hz}, 1 \mathrm{H}), 7.16$ (d, $J=7.5 \mathrm{~Hz}, 2 \mathrm{H}), 3.79$ (s, 3H), $3.72(\mathrm{~s}, 3 \mathrm{H}), 2.90(\mathrm{dd}, J=3.5,13.5 \mathrm{~Hz}, 1 \mathrm{H}), 2.65$ (dt, $J=2.5,13.5 \mathrm{~Hz}, 1 \mathrm{H}), 2.44$ (dt, $J=$ 3.0, $12.0 \mathrm{~Hz}, 1 \mathrm{H}), 2.34(\mathrm{dt}, J=2.5,14.0 \mathrm{~Hz}, 1 \mathrm{H}), 2.26(\mathrm{dd}, J=10.0,13.5 \mathrm{~Hz}, 1 \mathrm{H}), 1.90-$ 
$1.98(\mathrm{~m}, 2 \mathrm{H}), 1.62-1.70(\mathrm{~m}, 2 \mathrm{H}), 1.05(\mathrm{ddd}, J=3.0,12.0,24.0 \mathrm{~Hz}, 1 \mathrm{H}) ;{ }^{13} \mathrm{C}$ NMR $(125$ $\left.\mathrm{MHz}, \mathrm{CDCl}_{3}\right) \delta 181.5,172.2,171.2,139.7,129.5,128.5,126.4,54.5,53.1,53.0,46.6$, 41.0, 40.1, 34.2, 30.8, 27.0; LRMS (ESI+) Calcd. for $\mathrm{C}_{18} \mathrm{H}_{23} \mathrm{O}_{6}(\mathrm{M}+\mathrm{H})$ : 335. Found: 335.

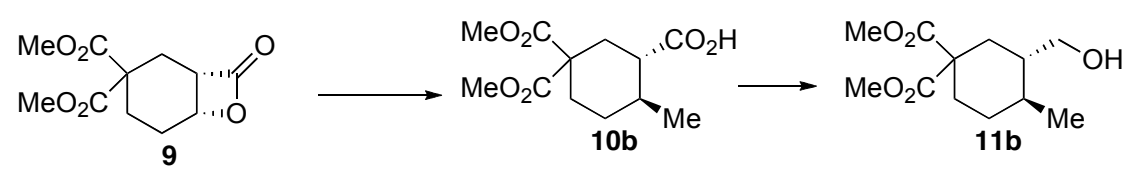

(+)-(3S,4S)-dimethyl 3-(hydroxymethyl)-4-methylcyclohexane-1,1-dicarboxylate 11b: A crude mixture of $\mathbf{1 0 b}$ was prepared according to the procedure $\mathbf{A}$ from CuBr•DMS (156 mg, $0.76 \mathrm{mmol}), \mathrm{Me}_{2} \mathrm{~S}$ (124 mg, $\left.0.15 \mathrm{~mL}, 2.0 \mathrm{mmol}\right), \mathrm{MeMgBr}(0.5$ $\mathrm{mL}, 1.5 \mathrm{mmol}, 3.0 \mathrm{M}$ in THF), bicyclic $\beta$-lactone 9 (60 mg, $0.25 \mathrm{mmol})$ and TMSCl (58 $\mathrm{mg}, 50 \mu \mathrm{L}, 0.4 \mathrm{mmol})$. To the above mixture in a solution of THF $(0.8 \mathrm{~mL})$ at $0^{\circ} \mathrm{C}$ was added a solution of $\mathrm{BH}_{3} \bullet$ THF $(0.40 \mathrm{~mL}, 0.40 \mathrm{mmol}, 1.0 \mathrm{M}$ in THF). After $1 \mathrm{~h}, \mathrm{MeOH}$ $(2.0 \mathrm{~mL})$ was added carefully. The solvent was removed in vacuo and purified by chromatography over silica gel, eluting with $20 \% \mathrm{EtOAc} / \mathrm{CH}_{2} \mathrm{Cl}_{2}$, to give $11 \mathbf{b}(32 \mathrm{mg}$, $0.14 \mathrm{mmol}, 54 \%)$ as a colorless oil: $[\alpha]_{\mathrm{D}}{ }^{23}+6.5\left(c 0.37, \mathrm{CHCl}_{3}\right)$; IR (thin film) 3432 (br), 2947, 1733, 1458, 1242, $1137 \mathrm{~cm}^{-1}$; ${ }^{1} \mathrm{H}$ NMR $\left(500 \mathrm{MHz}, \mathrm{CDCl}_{3}\right) \delta 3.77$ (s, 3H), 3.72$3.75(\mathrm{~m}, 1 \mathrm{H}), 3.72(\mathrm{~s}, 3 \mathrm{H}), 3.59-3.63(\mathrm{~m}, 1 \mathrm{H}), 2.45(\mathrm{dt}, J=2.5,13.5 \mathrm{~Hz}, 1 \mathrm{H}), 2.36$ (ddd, $J=3.0,5.5,13.5 \mathrm{~Hz}, 1 \mathrm{H}), 1.62-1.75(\mathrm{~m}, 3 \mathrm{H}), 1.27-1.35(\mathrm{~m}, 2 \mathrm{H}), 1.10-1.18(\mathrm{~m}, 1 \mathrm{H}), 0.94$ $(\mathrm{d}, J=6.0 \mathrm{~Hz}, 3 \mathrm{H}) ;{ }^{13} \mathrm{C} \mathrm{NMR}\left(125 \mathrm{MHz}, \mathrm{CDCl}_{3}\right) \delta$ 173.1, 171.9, 65.3, 55.4, 52.9, 52.7, 42.8, 34.5, 32.6, 31.9, 31.3, 19.7; LRMS (ESI+) Calcd. for $\mathrm{C}_{12} \mathrm{H}_{21} \mathrm{O}_{5}(\mathrm{M}+\mathrm{H})$ : 245. Found: 245.

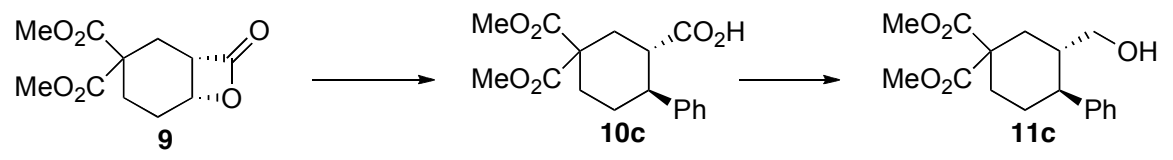

(+)-(3S,4S)-dimethyl 3-(hydroxymethyl)-4-phenylcyclohexane-1,1-dicarboxylate 11c: A crude mixture of $\mathbf{1 0 c}$ was prepared according to the procedure $\mathbf{C}$ from $\mathrm{CuCl}(91$ $\mathrm{mg}, 0.90 \mathrm{mmol}$ ), $\mathrm{Me}_{2} \mathrm{~S}$ (91 mg, $0.11 \mathrm{~mL}, 1.46 \mathrm{mmol}$ ), $\mathrm{PhMgCl}$ (0.90 mL, $1.8 \mathrm{mmol}, 2.0$ 
$\mathrm{M}$ in THF), bicyclic $\beta$-lactone 9 (44 mg, $0.18 \mathrm{mmol}$ ) and TMSCl (58 mg, $50 \mu \mathrm{L}, 0.4$ mmol). To the above mixture in a solution of THF $(0.8 \mathrm{~mL})$ at $0^{\circ} \mathrm{C}$ was added a solution of $\mathrm{BH}_{3} \cdot \mathrm{THF}(0.40 \mathrm{~mL}, 0.40 \mathrm{mmol}, 1.0 \mathrm{M}$ in THF). After $1 \mathrm{~h}, \mathrm{MeOH}(2.0 \mathrm{~mL})$ was added carefully. The solvent was removed in vacuo and purified by chromatography over silica gel, eluting with $20 \% \mathrm{EtOAc} / \mathrm{CH}_{2} \mathrm{Cl}_{2}$, to give $11 \mathrm{c}$ (37 $\mathrm{mg}, 0.12 \mathrm{mmol}, 67 \%$ ) as a colorless oil: $[\alpha]_{\mathrm{D}}{ }^{23}+4.9\left(c 0.41, \mathrm{CHCl}_{3}\right)$; IR (thin film) 3319 (br), 2959, 1729, 1456, 1258, $1090 \mathrm{~cm}^{-1} ;{ }^{1} \mathrm{H}$ NMR (500 MHz, $\mathrm{CDCl}_{3}$ ) $\delta$ 7.16-7.32 (m, 5H), 3.84 (s, 3H), 3.75 (s, $3 \mathrm{H}), 3.42(\mathrm{dd}, \mathrm{J}=3.5,11.0 \mathrm{~Hz}, 1 \mathrm{H}), 3.28(\mathrm{dd}, \mathrm{J}=5.5,11.0 \mathrm{~Hz}, 1 \mathrm{H}), 2.59(\mathrm{dt}, \mathrm{J}=3.0$, $14.0 \mathrm{~Hz}, 1 \mathrm{H}), 2.40-2.58(\mathrm{~m}, 2 \mathrm{H}), 1.90-1.98(\mathrm{~m}, 1 \mathrm{H}), 1.82-1.87(\mathrm{~m}, 2 \mathrm{H}), 1.74(\mathrm{t}, \mathrm{J}=13.0$ $\mathrm{Hz}, 1 \mathrm{H}), 1.62-1.68(\mathrm{~m}, 1 \mathrm{H}) ;{ }^{13} \mathrm{C}$ NMR $\left(125 \mathrm{MHz}, \mathrm{CDCl}_{3}\right) \delta 172.8,172.1,144.6,128.9$, 127.6, 126.8, 65.6, 55.2, 53.0, 52.9, 45.8, 41.6, 34.6, 31.6, 31.5; LRMS (ESI+) Calcd. for $\mathrm{C}_{17} \mathrm{H}_{23} \mathrm{O}_{5}(\mathrm{M}+\mathrm{H}): 307$. Found: 307.

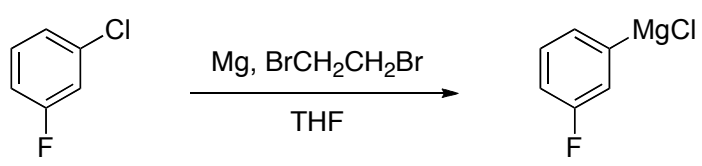

To a sealed tube equipped with a stir bar was added Mg turnings (730 mg, 30 mmol) and stirred vigorously under $\mathrm{N}_{2}$ for $12 \mathrm{~h}$. A solution of 3-fluorophenylchloride $(1.0 \mathrm{~g}, 0.8 \mathrm{~mL}, 7.5 \mathrm{mmol})$ and 1,2-dibromoethane (141 mg, $65 \mu \mathrm{L}, 0.75 \mathrm{mmol})$ in THF $(6.7 \mathrm{~mL})$ was added via cannula. The tube was quickly sealed and kept at $85-88^{\circ} \mathrm{C}$ for $2.5 \mathrm{~h}$ to give a dark tan solution of $m-\mathrm{F}-\mathrm{PhMgCl}(0.85 \mathrm{M}$ in THF $){ }^{4}$

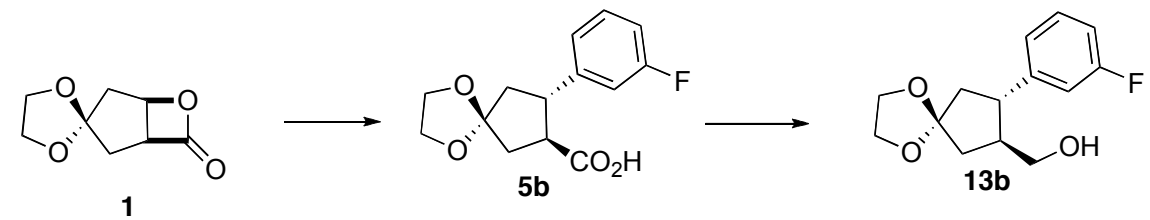

(+)-((7S,8S)-8-(3-fluorophenyl)-1,4-dioxaspiro[4.4]nonan-7-yl)methanol 13b: A crude mixture containing $\mathbf{5 b}$ and $\mathbf{7}$ was prepared according to the procedure $\mathbf{C}$ from $\mathrm{CuCl}$

\footnotetext{
${ }^{4}$ Titrated by no-D NMR spectroscopy method: Hoye, T. R.; Eklov, B. M.; Voloshin, M. Org. Lett. 2004, 6, 2567.
} 
(103 mg, $1.04 \mathrm{mmol}$ ), $\mathrm{Me}_{2} \mathrm{~S}$ (208 mg, $0.24 \mathrm{~mL}, 3.3 \mathrm{mmol}$ ), $m-\mathrm{F}-\mathrm{C}_{6} \mathrm{H}_{4} \mathrm{MgCl}$ in THF (2.45 $\mathrm{mL}, 2.1 \mathrm{mmol}, 0.85 \mathrm{M}$ in THF), bicyclic $\beta$-lactone 1 (35 mg, $0.20 \mathrm{mmol}$ ) and TMSCl (45 mg, $52 \mu \mathrm{L}, 0.6 \mathrm{mmol}$ ). To the above mixture in a solution of THF $(1.8 \mathrm{~mL})$ at $0^{\circ} \mathrm{C}$ was added a solution of $\mathrm{BH}_{3} \bullet$ THF $(0.40 \mathrm{~mL}, 0.40 \mathrm{mmol}, 1.0 \mathrm{M}$ in THF). After $1 \mathrm{~h}$, $\mathrm{MeOH}(2.0 \mathrm{~mL})$ was added carefully. The solvent was removed in vacuo and purified by chromatography over silica gel, eluting with $20 \%$ EtOAc $/ \mathrm{CH}_{2} \mathrm{Cl}_{2}$, to give $\mathbf{1 3 b}(32 \mathrm{mg}$, $0.12 \mathrm{mmol}, 64 \%)$ as a colorless oil: $[\alpha]_{\mathrm{D}}^{23}+12.9\left(c\right.$ 1.24, $\left.\mathrm{CHCl}_{3}\right)$; IR (thin film) 3404 (br), 2925, 1615, 1590, 1448, 1321, 1135, $1078 \mathrm{~cm}^{-1} ;{ }^{1} \mathrm{H}$ NMR (500 MHz, $\left.\mathrm{CDCl}_{3}\right) \delta 7.28$ $(\mathrm{dt}, J=8.0,14.5 \mathrm{~Hz}, 1 \mathrm{H}), 7.06(\mathrm{~d}, J=7.5 \mathrm{~Hz}, 1 \mathrm{H}), 7.01(\mathrm{dt}, J=2.0,10.0 \mathrm{~Hz}, 1 \mathrm{H}), 6.92$ (ddt, $J=1.0,2.0,9.0 \mathrm{~Hz}, 1 \mathrm{H}), 3.93-4.03(\mathrm{~m}, 4 \mathrm{H}), 3.67$ (dd, $J=4.0,10.5 \mathrm{~Hz}, 1 \mathrm{H}), 3.54$ (dd, $J=6.0,10.5 \mathrm{~Hz}, 1 \mathrm{H}), 3.02(\mathrm{dd}, J=10.5,19.0 \mathrm{~Hz}, 1 \mathrm{H}), 2.26-2.37$ (m, 3H), 2.05 (ddd, $J=1.0,11.0,13.5 \mathrm{~Hz}, 1 \mathrm{H}), 1.87$ (ddd, $J=1.0,9.0,13.0 \mathrm{~Hz}, 1 \mathrm{H}) ;{ }^{13} \mathrm{C} \mathrm{NMR}(125 \mathrm{MHz}$, $\left.\mathrm{CDCl}_{3}\right) \delta 163.3\left(\mathrm{~d}, J_{\mathrm{C}-\mathrm{F}}=244.0 \mathrm{~Hz}\right), 146.8\left(\mathrm{~d}, J_{\mathrm{C}-\mathrm{F}}=6.8 \mathrm{~Hz}\right), 130.2\left(\mathrm{~d}, J_{\mathrm{C}-\mathrm{F}}=8.4 \mathrm{~Hz}\right)$, $123.4\left(\mathrm{~d}, J_{\mathrm{C}-\mathrm{F}}=2.3 \mathrm{~Hz}\right), 116.2,114.5\left(\mathrm{~d}, J_{\mathrm{C}-\mathrm{F}}=21.3 \mathrm{~Hz}\right), 113.6\left(\mathrm{~d}, J_{\mathrm{C}-\mathrm{F}}=20.5 \mathrm{~Hz}\right), 64.76$, 64.73, 64.6, 47.8, $45.5\left(\mathrm{~d}, J_{\mathrm{C}-\mathrm{F}}=1.5 \mathrm{~Hz}\right), 45.3,39.9$; LRMS (CI+) Calcd. for $\mathrm{C}_{14} \mathrm{H}_{18} \mathrm{O}_{3} \mathrm{~F}(\mathrm{M}+\mathrm{H}): 253$. Found: 253.

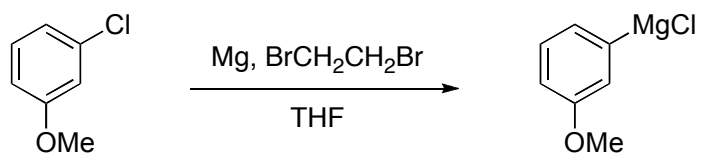

To a sealed tube equipped with a stir bar was added $\mathrm{Mg}$ turnings (730 $\mathrm{mg}, 30$ mmol). It was stirred vigorously under $\mathrm{N}_{2}$ for $12 \mathrm{~h}$. A solution of 3methoxyphenylchloride $(1.1 \mathrm{~g}, 0.91 \mathrm{~mL}, 7.5 \mathrm{mmol})$ and 1,2-dibromoethane $(141 \mathrm{mg}, 65$ $\mu \mathrm{L}, 0.75 \mathrm{mmol})$ in THF $(6.7 \mathrm{~mL})$ was added via cannula. The tube was quickly sealed and kept at $85-88^{\circ} \mathrm{C}$ for $5 \mathrm{~h}$ to give a dark tan solution of $m$-OMe-PhMgCl $(0.95 \mathrm{M}$ in THF).

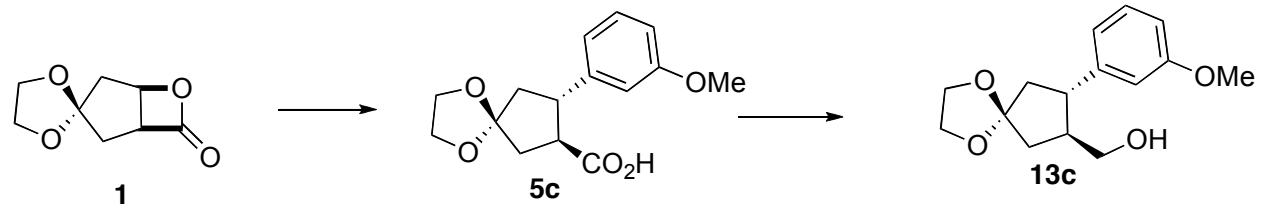

(+)-((7S,8S)-8-(3-methoxyphenyl)-1,4-dioxaspiro[4.4]nonan-7-yl)methanol 13c: A crude mixture containing $\mathbf{5 c}$ and $\mathbf{7}$ was prepared according to the procedure $\mathbf{C}$ from 
$\mathrm{CuCl}(99 \mathrm{mg}, 1.0 \mathrm{mmol}), \mathrm{Me}_{2} \mathrm{~S}(284 \mathrm{mg}, 0.24 \mathrm{~mL}, 4.8 \mathrm{mmol}), m-\mathrm{OMe}-\mathrm{C}_{6} \mathrm{H}_{4} \mathrm{MgCl}$ and bicyclic $\beta$-lactone 1 (35 mg, $0.20 \mathrm{mmol}$ ) and TMSCl (58 mg, $50 \mu \mathrm{L}, 0.4 \mathrm{mmol}$ ). To the above mixture in a solution of THF $(1.8 \mathrm{~mL})$ at $0^{\circ} \mathrm{C}$ was added a solution of $\mathrm{BH}_{3} \cdot \mathrm{THF}$ $(0.40 \mathrm{~mL}, 0.40 \mathrm{mmol}, 1.0 \mathrm{M}$ in THF). After $1 \mathrm{~h}, \mathrm{MeOH}(2.0 \mathrm{~mL})$ was added carefully. The solvent was removed in vacuo and purified by chromatography over silica gel, eluting with $20 \%$ EtOAc $/ \mathrm{CH}_{2} \mathrm{Cl}_{2}$, to give $13 \mathrm{c}$ (27 mg, $0.10 \mathrm{mmol}, 52 \%$ ) as a colorless oil: $[\alpha]_{\mathrm{D}}^{23}+8.8\left(c 1.13, \mathrm{CHCl}_{3}\right)$; IR (thin film) 3420 (br), 2941, 1611, 1490, 1437, 1321, $1257 \mathrm{~cm}^{-1} ;{ }^{1} \mathrm{H}$ NMR $\left(500 \mathrm{MHz}, \mathrm{CDCl}_{3}\right) \delta 7.23(\mathrm{t}, J=7.5 \mathrm{~Hz}, 1 \mathrm{H}), 6.87(\mathrm{~d}, J=7.5 \mathrm{~Hz}$, $1 \mathrm{H}), 6.84(\mathrm{t}, J=1.5 \mathrm{~Hz}, 1 \mathrm{H}), 6.77$ (dd, $J=2.7,8.4 \mathrm{~Hz}, 1 \mathrm{H}), 3.81-4.00(\mathrm{~m}, 4 \mathrm{H}), 3.78$ (s, $3 \mathrm{H}), 3.64(\mathrm{dd}, J=4.0,11.0 \mathrm{~Hz}, 1 \mathrm{H}), 3.51(\mathrm{dd}, J=6.0,10.5 \mathrm{~Hz}, 1 \mathrm{H}), 2.96(\mathrm{dt}, J=8.0$, $10.0 \mathrm{~Hz}, 1 \mathrm{H}), 2.27-2.39$ (m, 3H), 2.07 (dd, $J=11.5,13.5 \mathrm{~Hz}, 1 \mathrm{H}), 1.86$ (ddd, $J=1.5,9.0$, $13.5 \mathrm{~Hz}, 1 \mathrm{H}) ;{ }^{13} \mathrm{C} \mathrm{NMR}\left(125 \mathrm{MHz}, \mathrm{CDCl}_{3}\right) \delta 160.0,145.6,129.8,120.1,116.4,113.6$, 111.9, 64.9, 64.7, 64.5, 55.4, 47.8, 46.0, 45.4, 40.0; LRMS (ESI+) Calcd. for $\mathrm{C}_{15} \mathrm{H}_{20} \mathrm{O}_{4} \mathrm{Li}(\mathrm{M}+\mathrm{Li}): 271$. Found: 271.

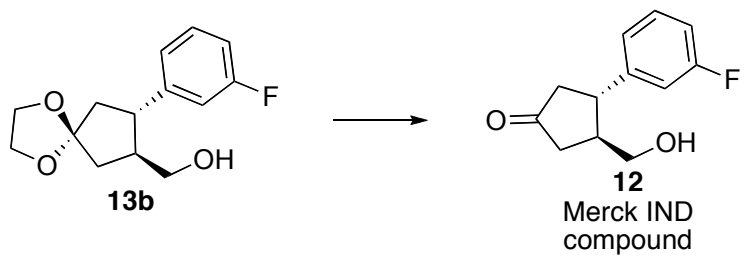

(+)-(3S,4S)-3-(3-fluorophenyl)-4-(hydroxymethyl)cyclopentanone 12: To a solution of $13 \mathbf{b}(26 \mathrm{mg}, 0.1 \mathrm{mmol})$ in $\mathrm{THF}(2.4 \mathrm{~mL})$ at $23^{\circ} \mathrm{C}$ was added $1 \mathrm{~N} \mathrm{HCl}$ solution $(0.6 \mathrm{~mL}, 0.6 \mathrm{mmol})$. After $16 \mathrm{~h}$, the reaction was quenched with sat. aq. $\mathrm{NaCl}$ solution $(10 \mathrm{~mL})$. The mixture was extracted with EtOAc $(3 \mathrm{X} 10 \mathrm{~mL})$. The dried extract $\left(\mathrm{MgSO}_{4}\right)$ was concentrated in vacuo and purified by chromatography over silica gel, eluting with $20 \% \mathrm{EtOAc} / \mathrm{CH}_{2} \mathrm{Cl}_{2}$, to give Merck IND compound 12 (21 mg, $0.96 \mathrm{mmol}$, $95 \%)$ as a colorless oil: $[\alpha]_{\mathrm{D}}^{23}+75.3\left(c 1.24, \mathrm{CHCl}_{3}\right)^{5}$ Other data matched that previously reported: ${ }^{6}$ IR (thin film) 3423 (br), 2925, 1742, 1589, 1488, $1148 \mathrm{~cm}^{-1}$; ${ }^{1} \mathrm{H}$ NMR (500

\footnotetext{
${ }^{5}$ Rotation data for this Merck IND intermediate was not reported in either the primary or patent literature (see ref. 5 below). Based on the \%ee of the starting $\beta$-lactone 1 (determined to be $92 \%$ ee by chiral GC analysis), the enantiomeric purity of cyclopentanone 12 should be $\geq 92 \%$ ee.
}

${ }^{6}$ (a) Palucki, M.; Um, J. M.; Yasuda, N.; Conlon, D. A.; Tsay, F. R.; Hartner, F. W.; Hsiao, Y.; Marcune, B.; Karady, S.; Hughes, D. L.; Dormer, P. G.; Reider, P. J. J. Org. 
$\left.\mathrm{MHz}, \mathrm{CDCl}_{3}\right) \delta 7.33(\mathrm{dt}, J=8.0,14.0 \mathrm{~Hz}, 1 \mathrm{H}), 7.07(\mathrm{~d}, J=8.0 \mathrm{~Hz}, 1 \mathrm{H}), 6.96-7.01(\mathrm{~m}$, 2H), $3.74(\mathrm{dd}, J=4.0,11.0 \mathrm{~Hz}, 1 \mathrm{H}), 3.62(\mathrm{dd}, J=5.5,11.0 \mathrm{~Hz}, 1 \mathrm{H}), 3.29(\mathrm{dt}, J=8.0$, $11.0 \mathrm{~Hz}, 1 \mathrm{H}), 2.76(\mathrm{dd}, J=8.0,19.0 \mathrm{~Hz}, 1 \mathrm{H}), 2.61(\mathrm{dd}, J=7.5,18.5 \mathrm{~Hz}, 1 \mathrm{H}), 2.48-2.53$ (m, 1H), 2.45 (ddd, $J=1.5,11.5,18.5 \mathrm{~Hz}, 1 \mathrm{H}), 2.36(\mathrm{ddd}, J=1.0,11.0,18.0 \mathrm{~Hz}, 1 \mathrm{H})$; ${ }^{13} \mathrm{C}$ NMR $\left(125 \mathrm{MHz}, \mathrm{CDCl}_{3}\right) \delta 216.3,163.4\left(\mathrm{~d}, J_{\mathrm{C}-\mathrm{F}}=245.4 \mathrm{~Hz}\right), 144.7\left(\mathrm{~d}, J_{\mathrm{C}-\mathrm{F}}=6.9 \mathrm{~Hz}\right)$, $130.6\left(\mathrm{~d}, J_{\mathrm{C}-\mathrm{F}}=8.5 \mathrm{~Hz}\right), 123.2\left(\mathrm{~d}, J_{\mathrm{C}-\mathrm{F}}=2.6 \mathrm{~Hz}\right), 114.4\left(\mathrm{~d}, J_{\mathrm{C}-\mathrm{F}}=21.1 \mathrm{~Hz}\right), 114.2\left(\mathrm{~d}, J_{\mathrm{C}-\mathrm{F}}=\right.$ $21.3 \mathrm{~Hz}), 62.8,47.4,46.5,44.2\left(\mathrm{~d}, J_{\mathrm{C}-\mathrm{F}}=1.6 \mathrm{~Hz}\right), 42.0$; LRMS (CI+) Calcd. for $\mathrm{C}_{12} \mathrm{H}_{14} \mathrm{O}_{2} \mathrm{~F}(\mathrm{M}+\mathrm{H})$ : 209. Found: 209.

Chem. 2002, 67, 5508. (b) Conlon, D. A.; Jensen, M. S.; Plaucki, M.; Yasuda, N.; Um, J. M.; Yang, C.; Hartner, F. W.; Tsay, F.-R.; Hsiao, Y.; Pye, P.; Rivera, N. R.; Hughes, D. L. Chirality 2005, 17, S149. 


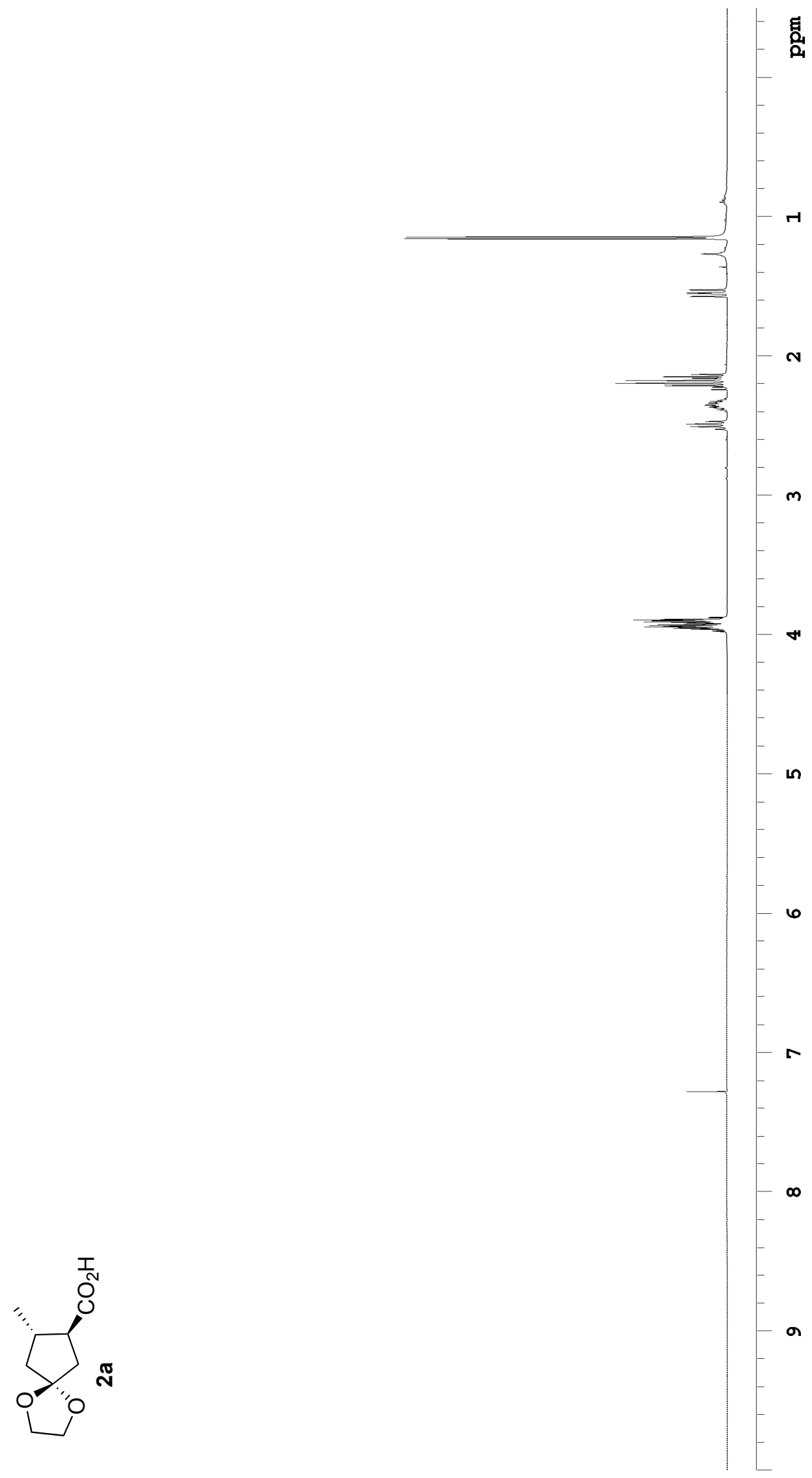




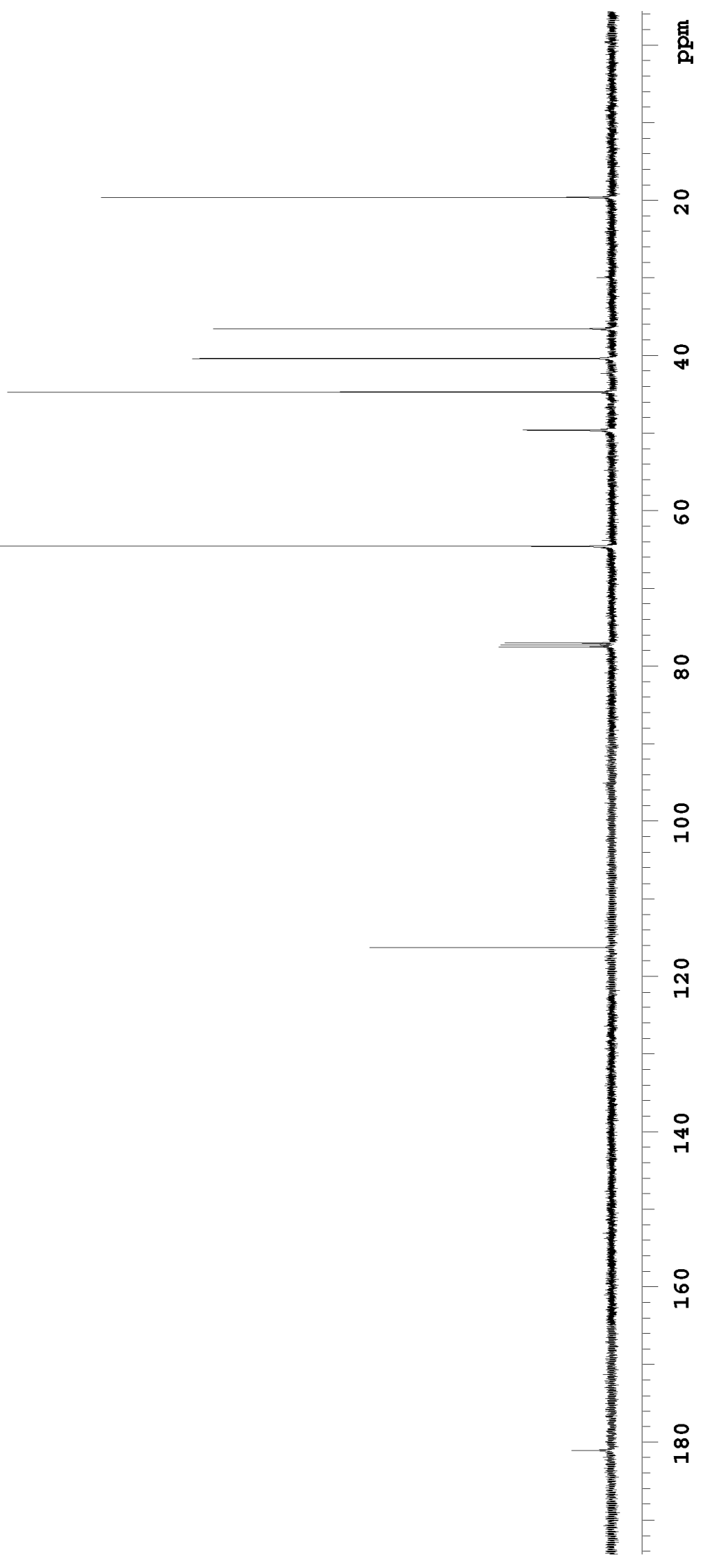




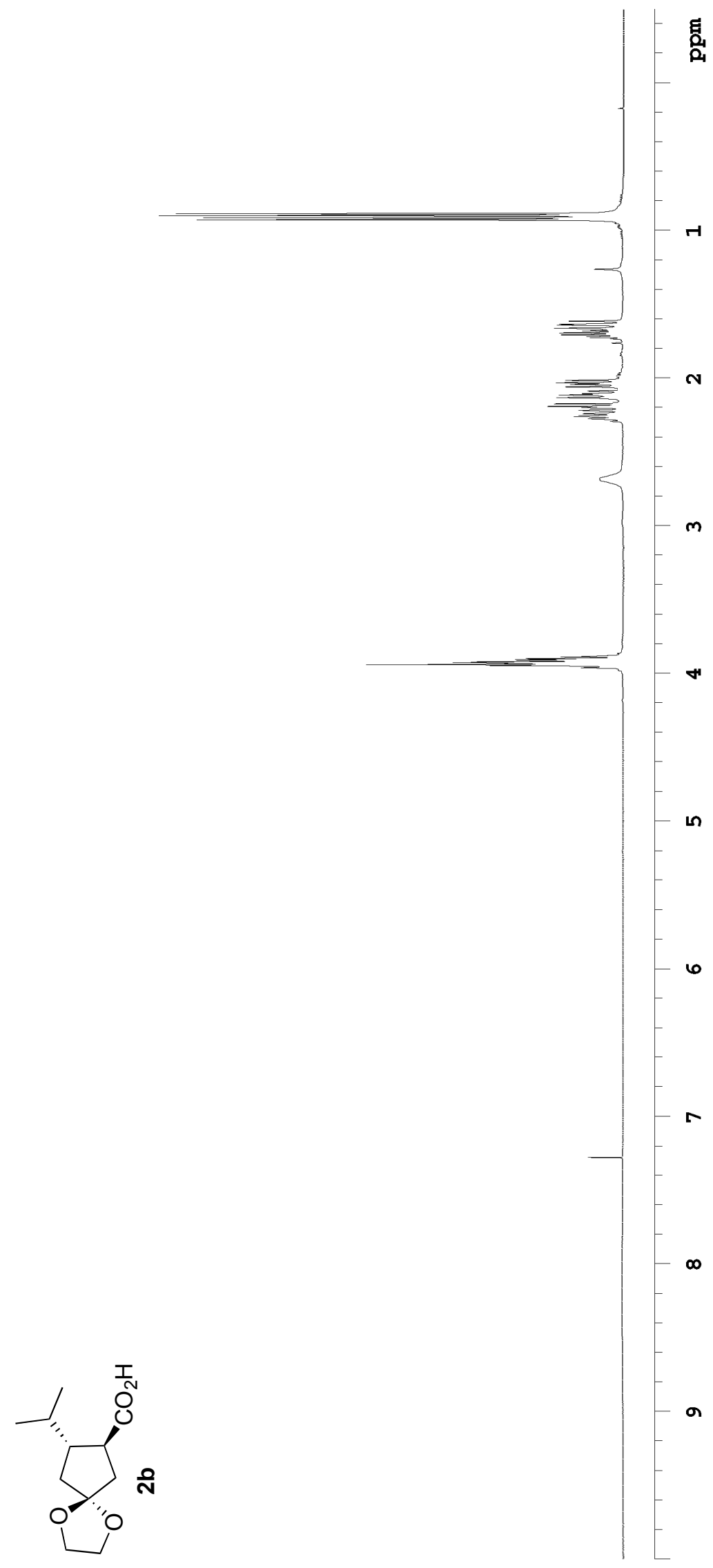




$$
1
$$




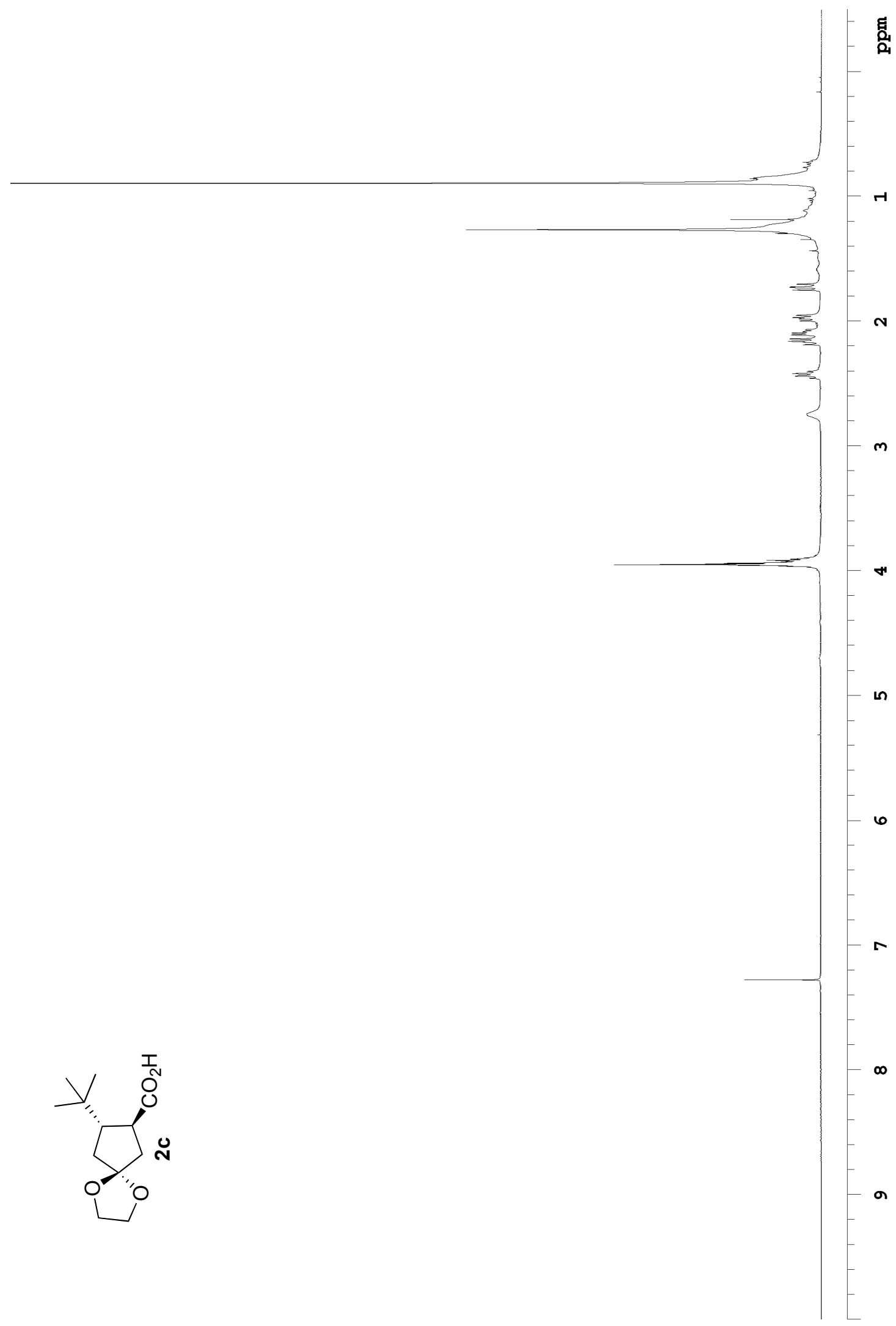




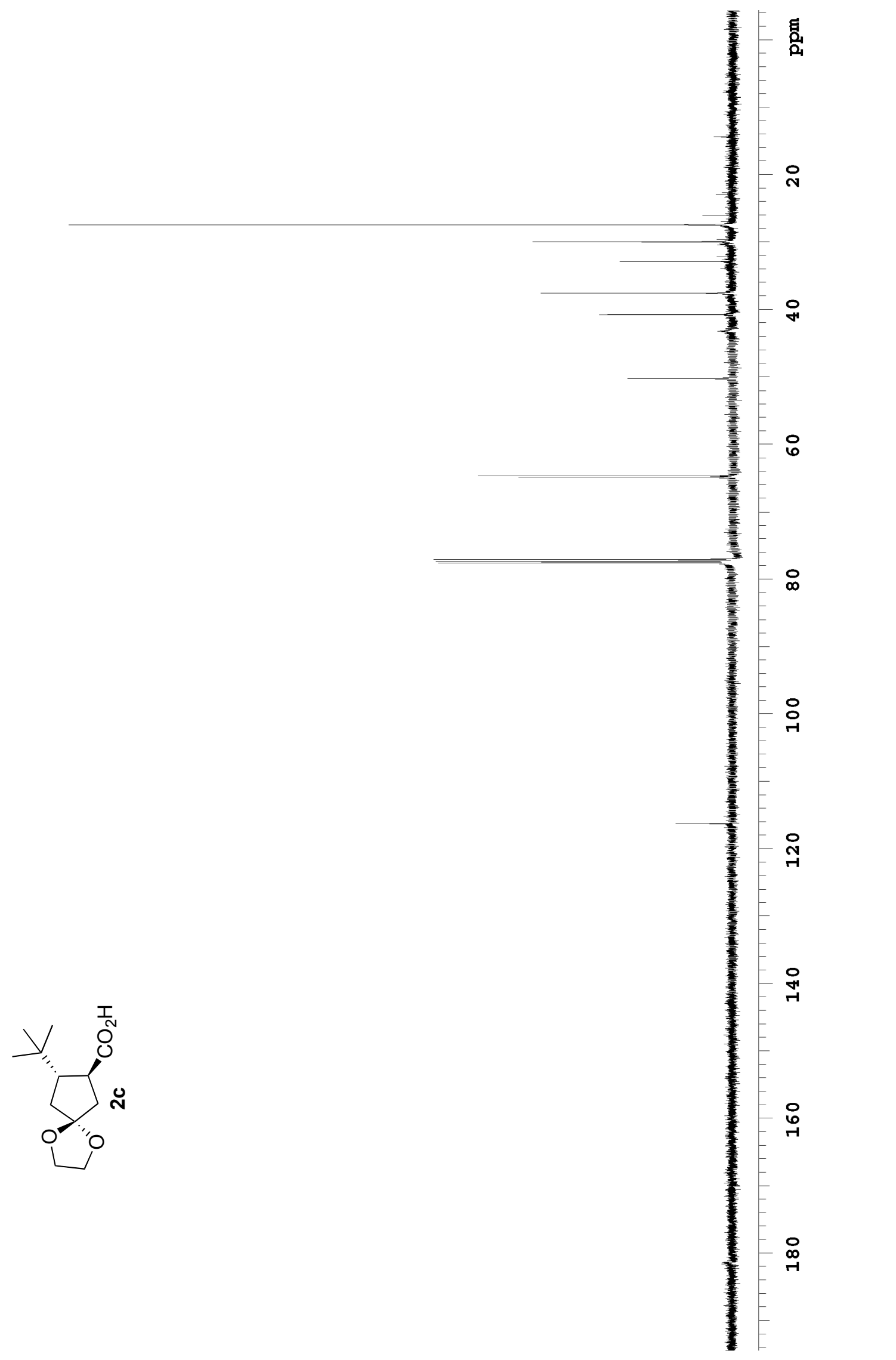




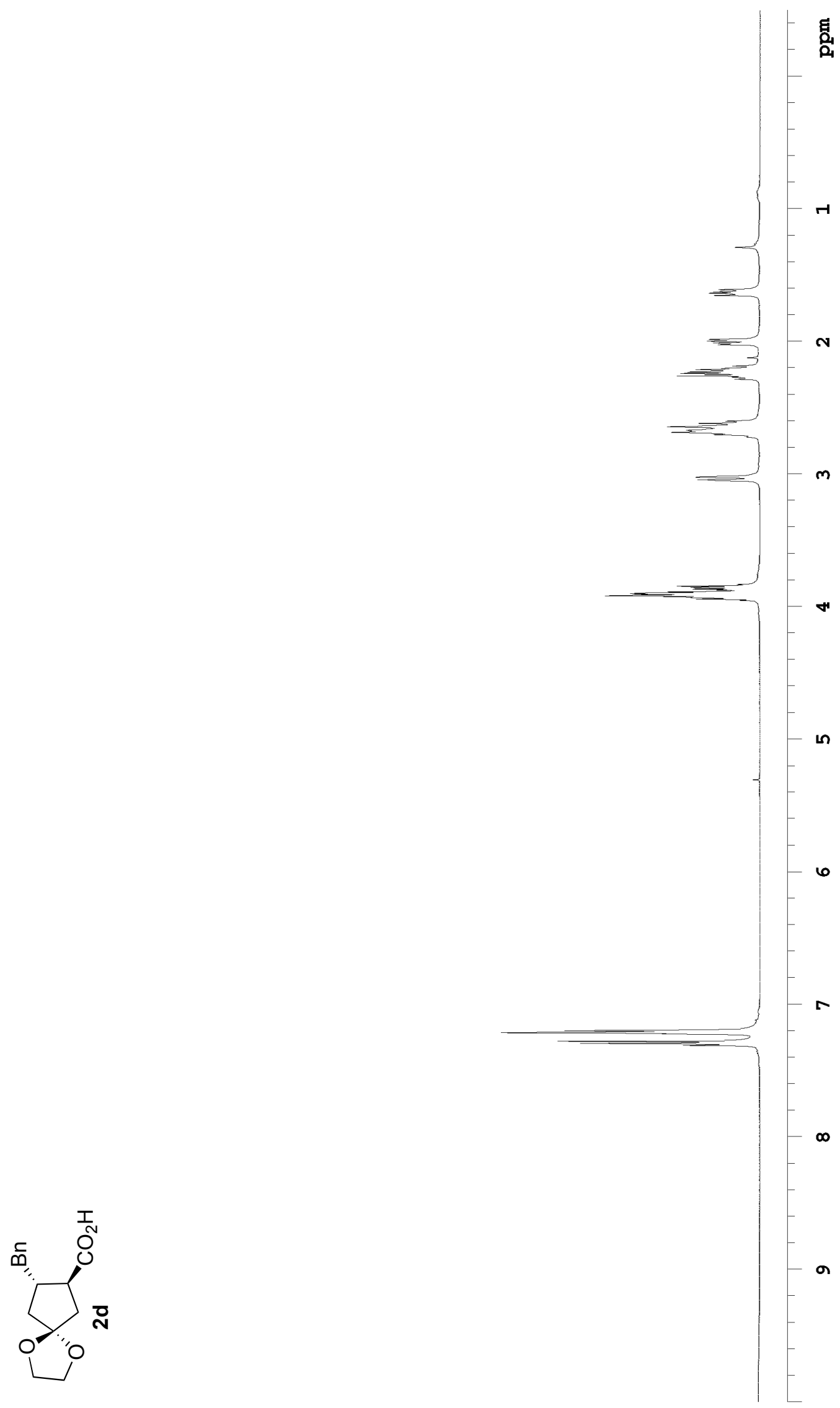




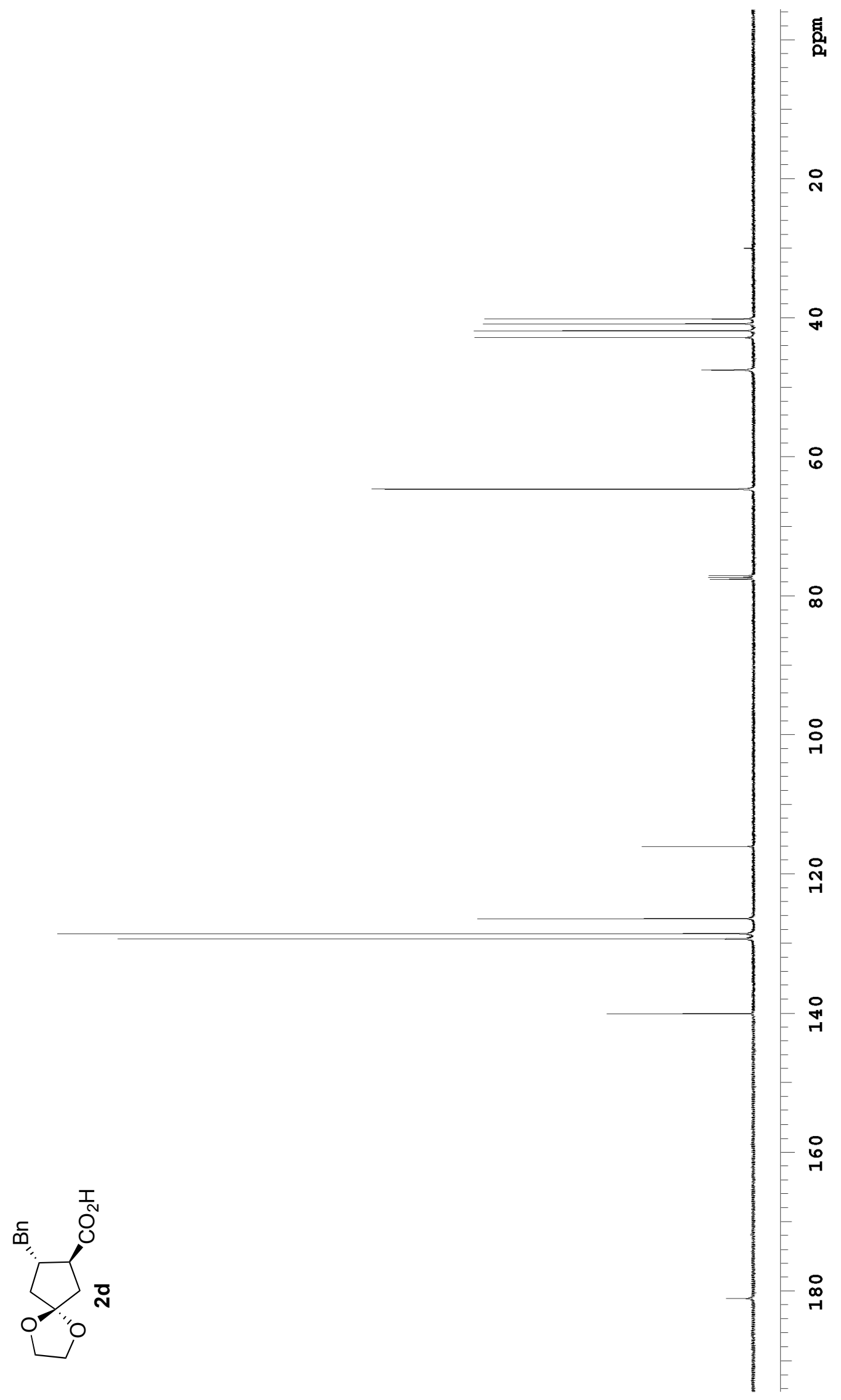




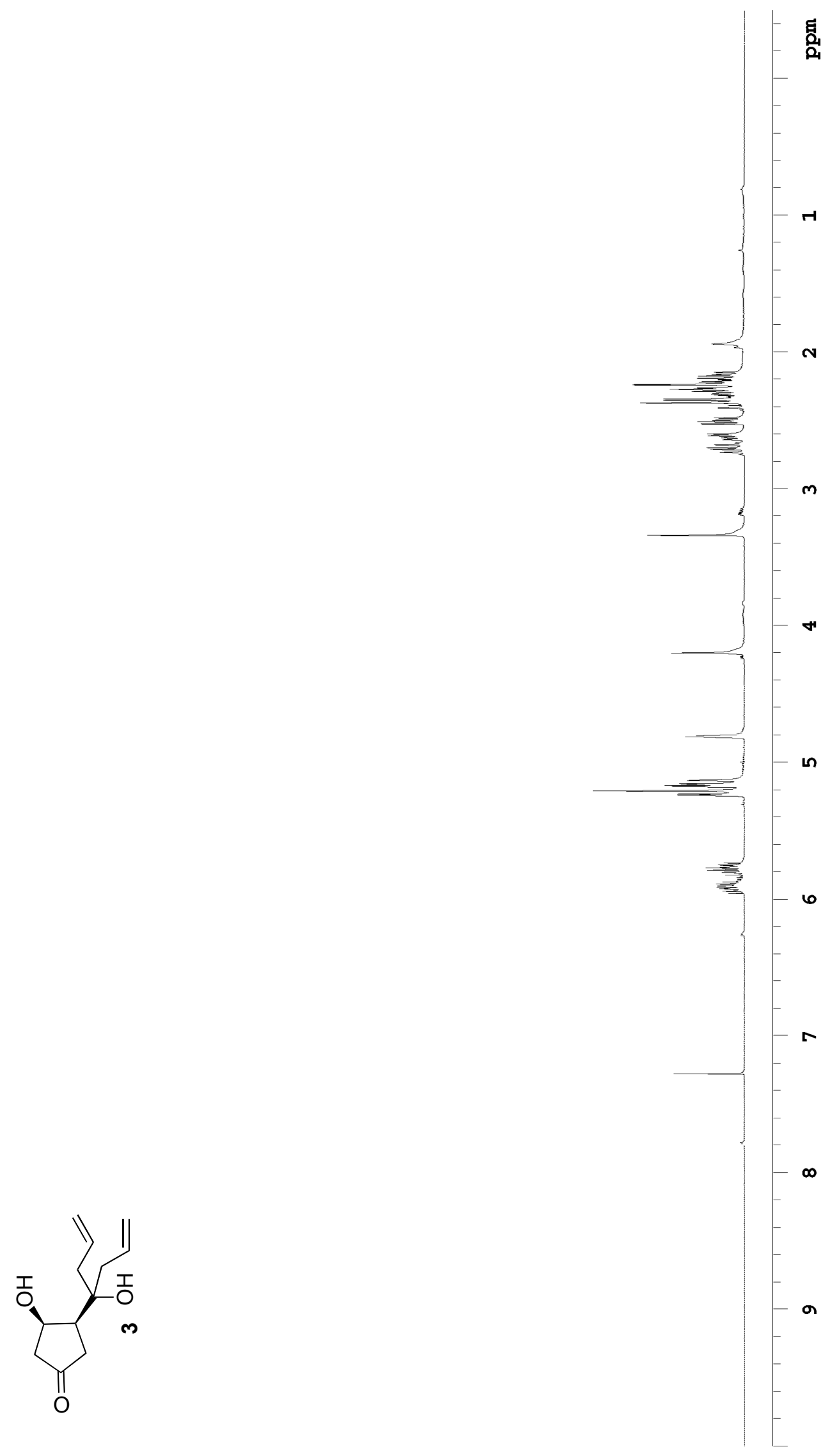




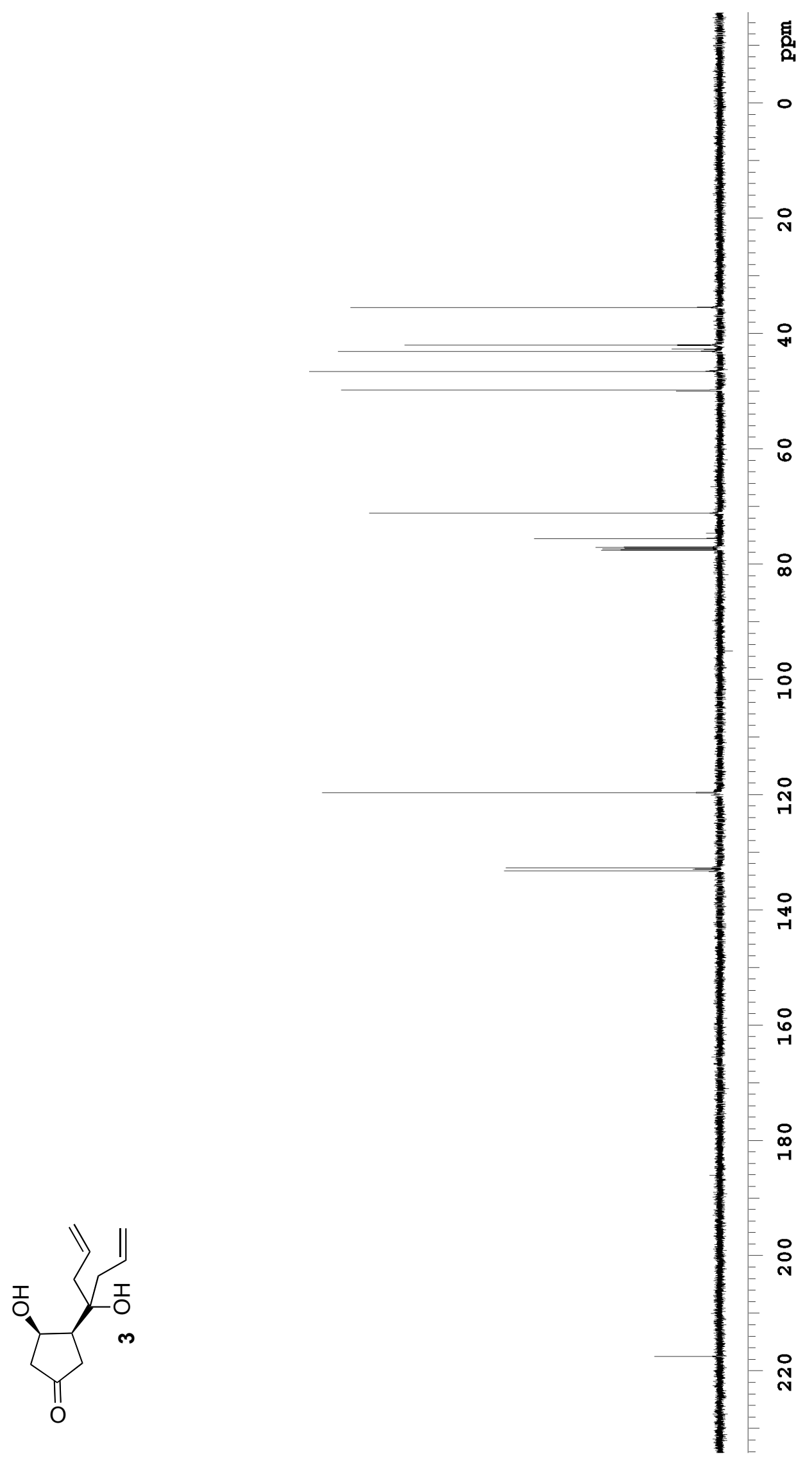




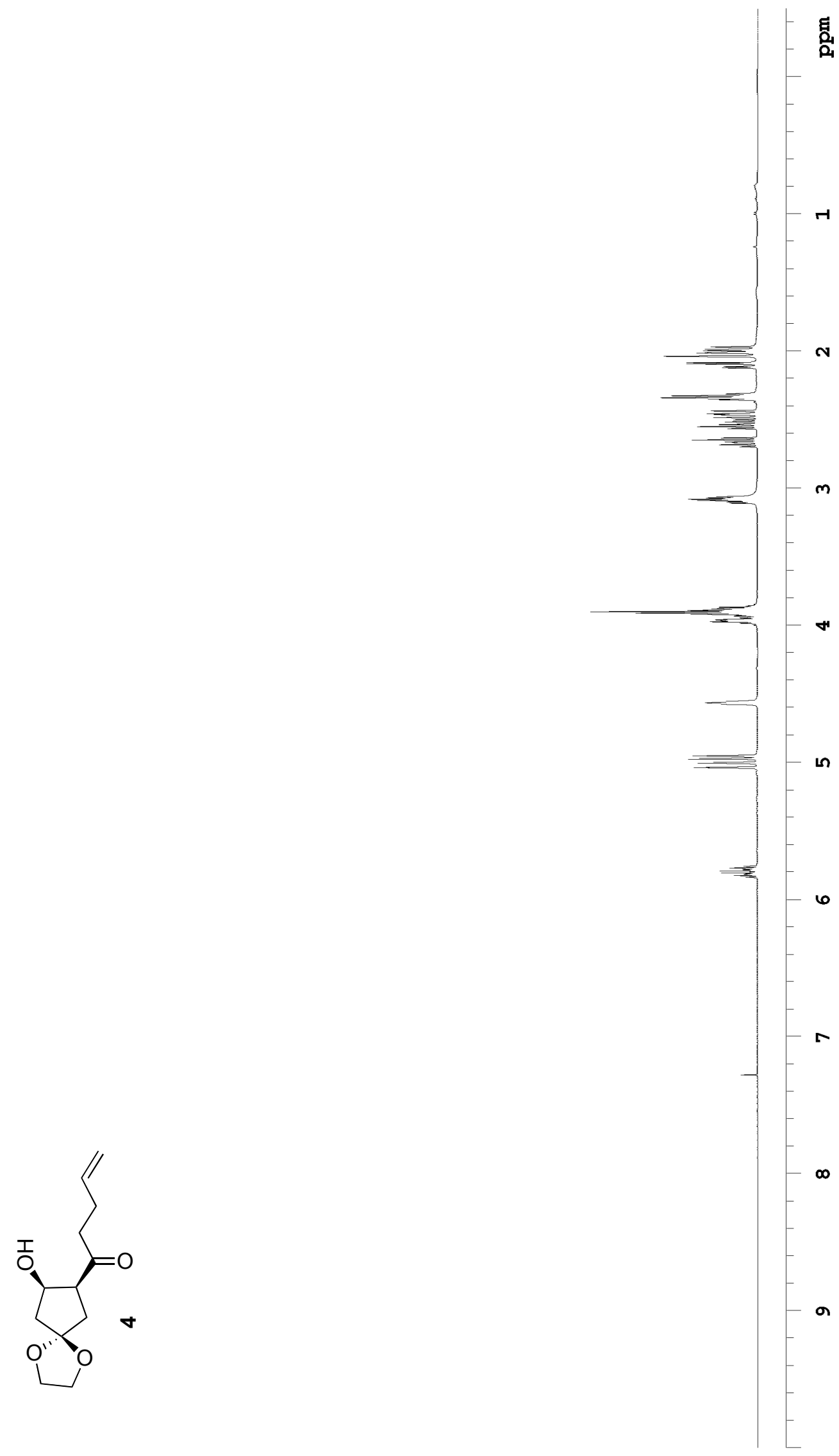




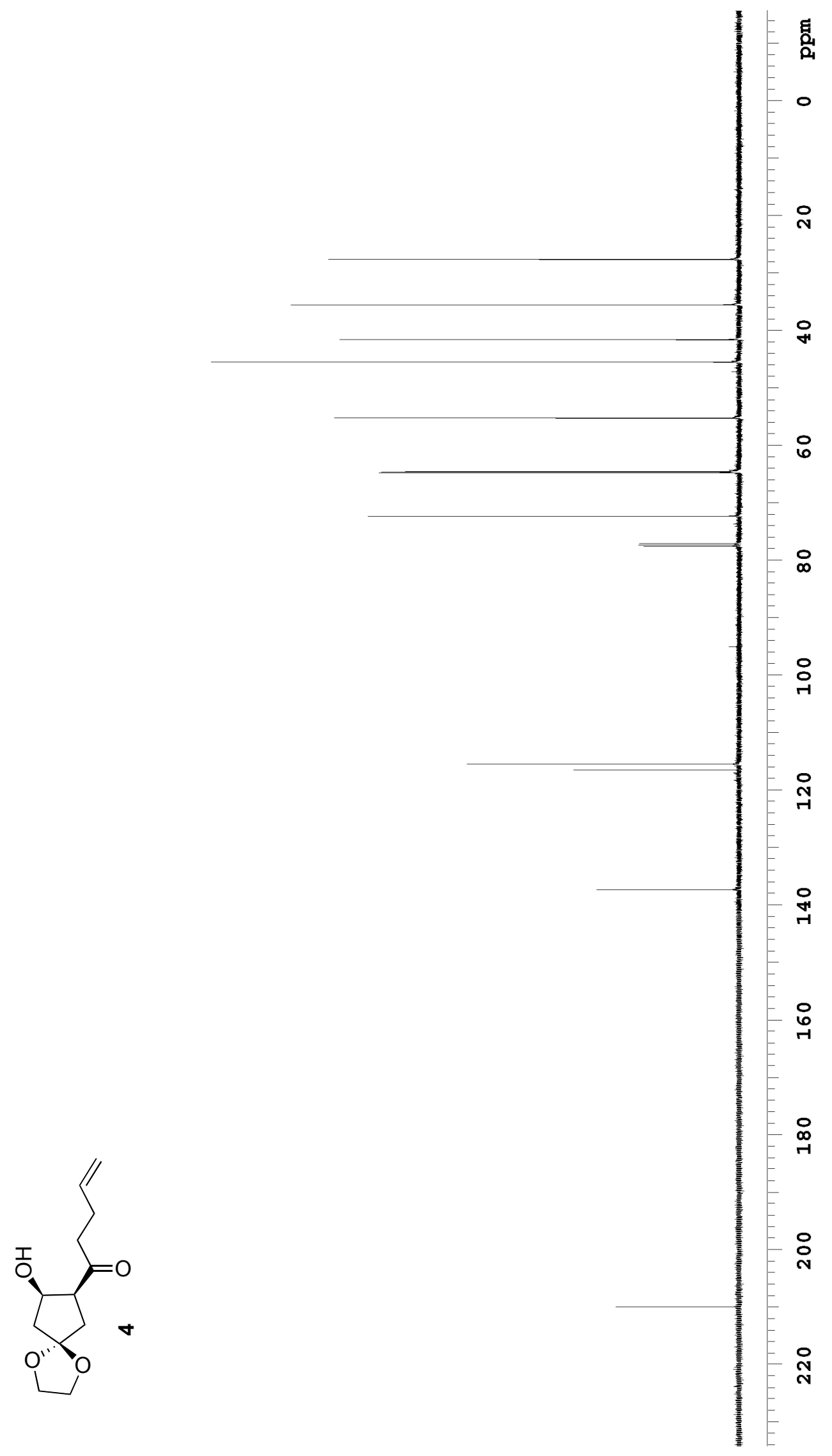




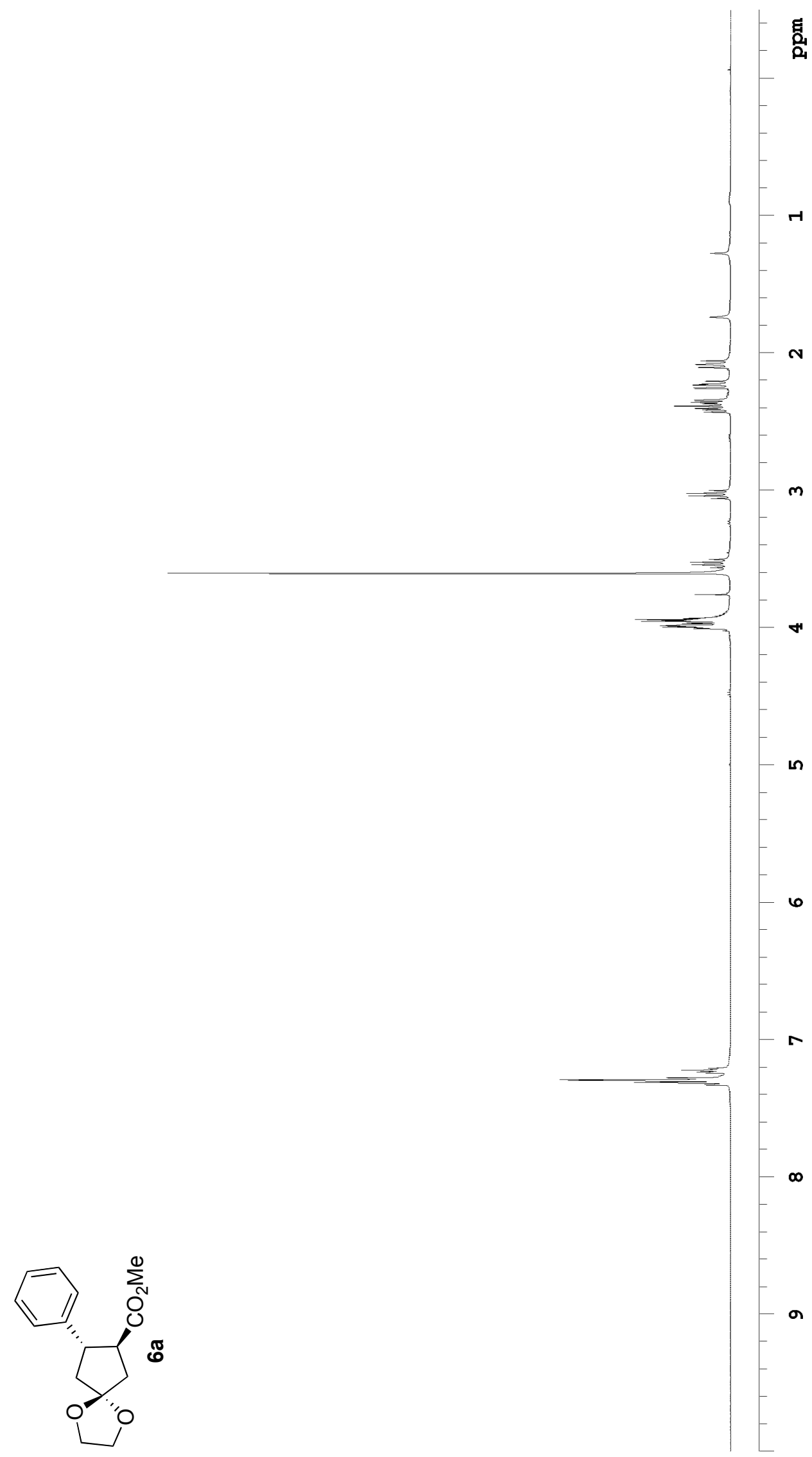




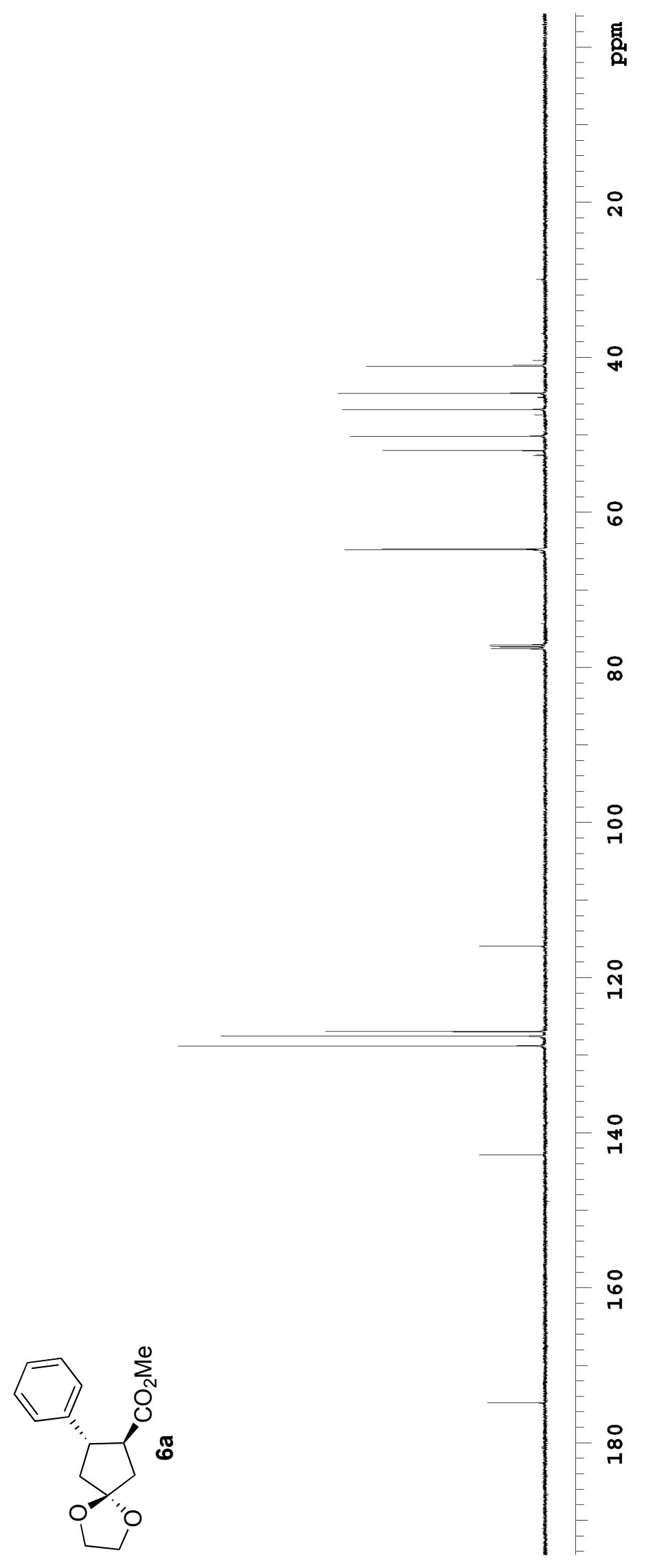




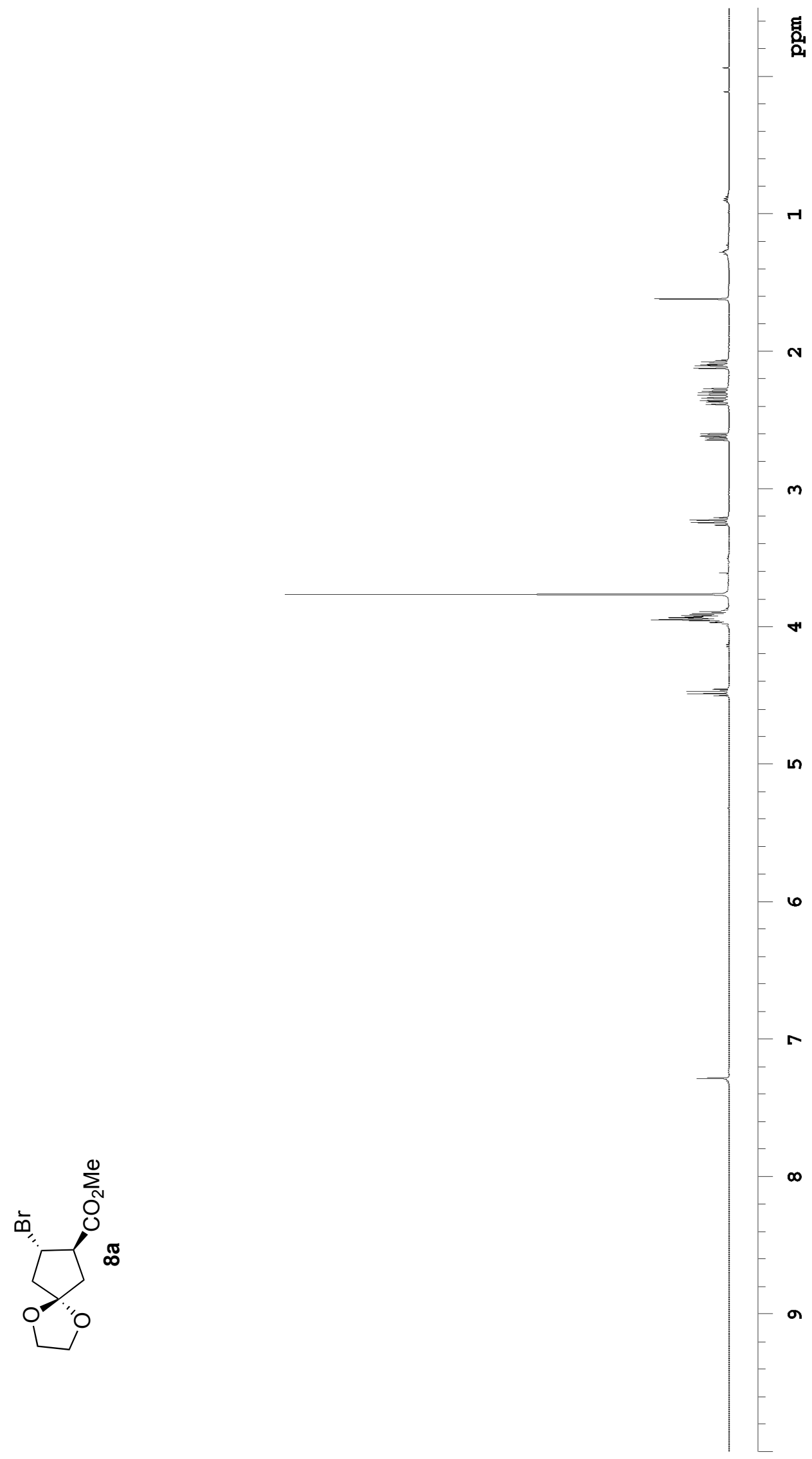




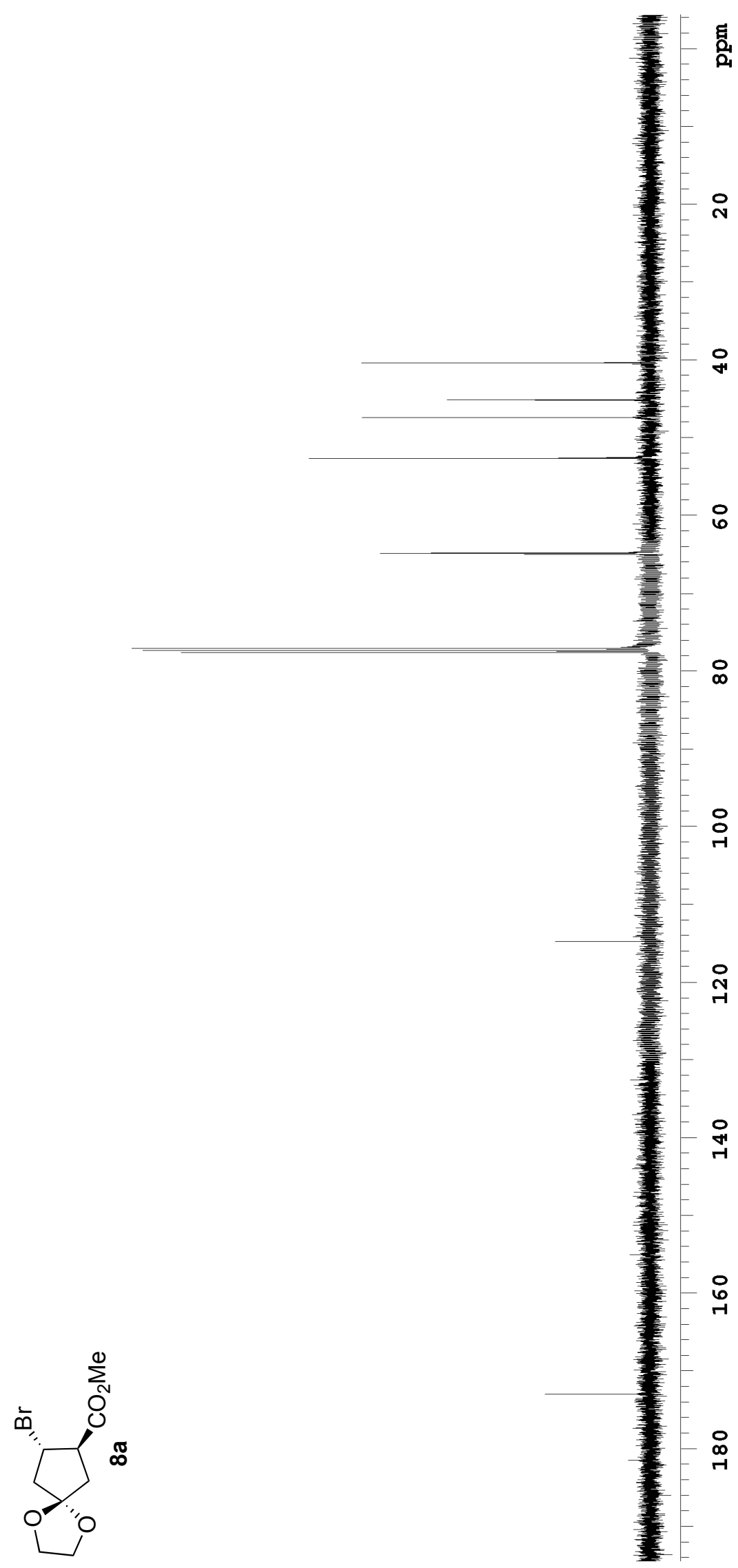




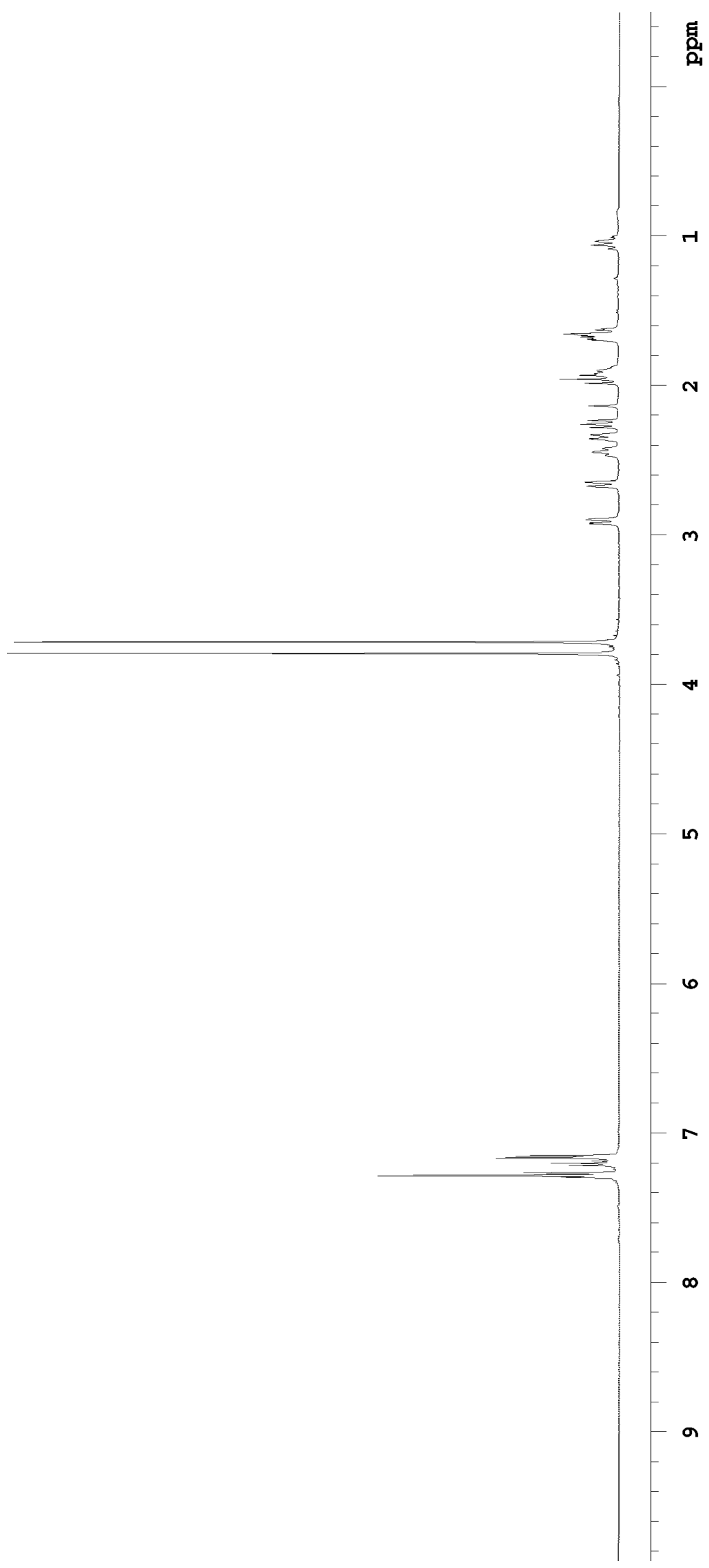




$$
\text { . }
$$




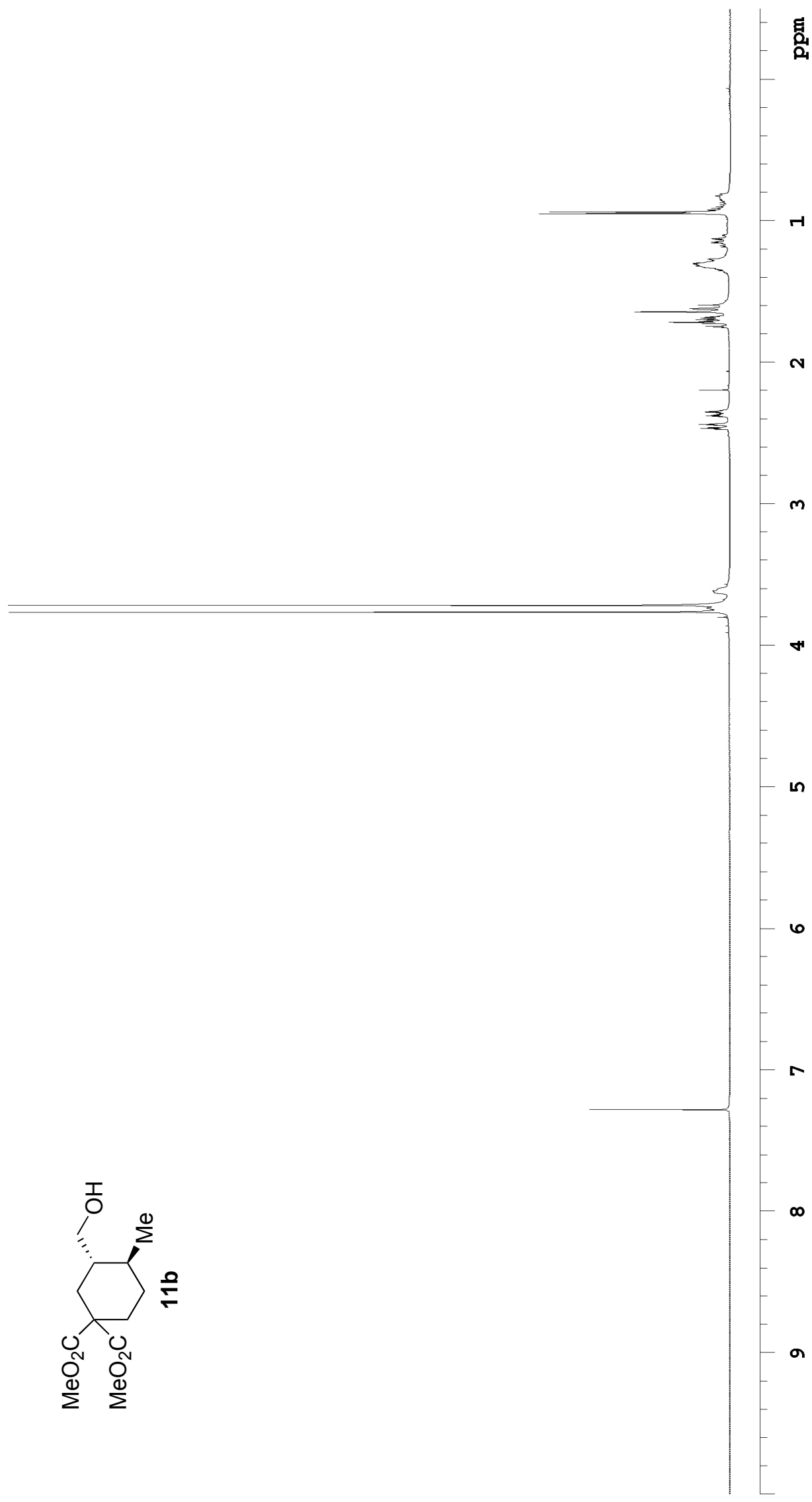


S33

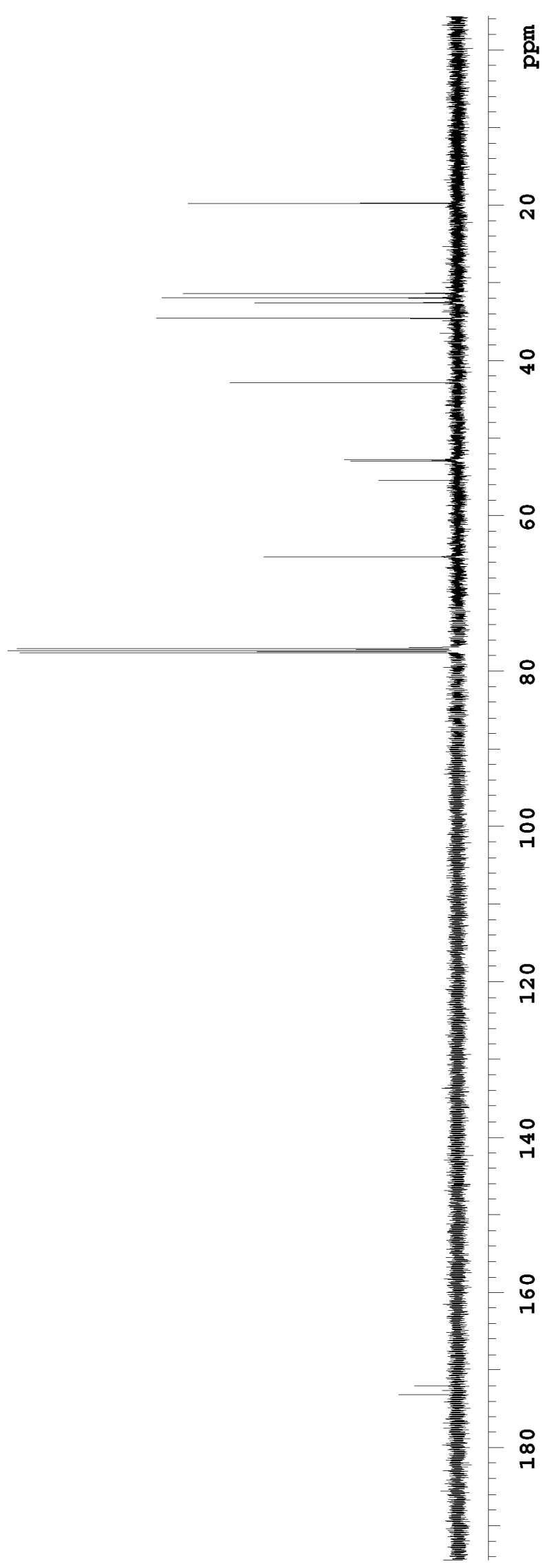




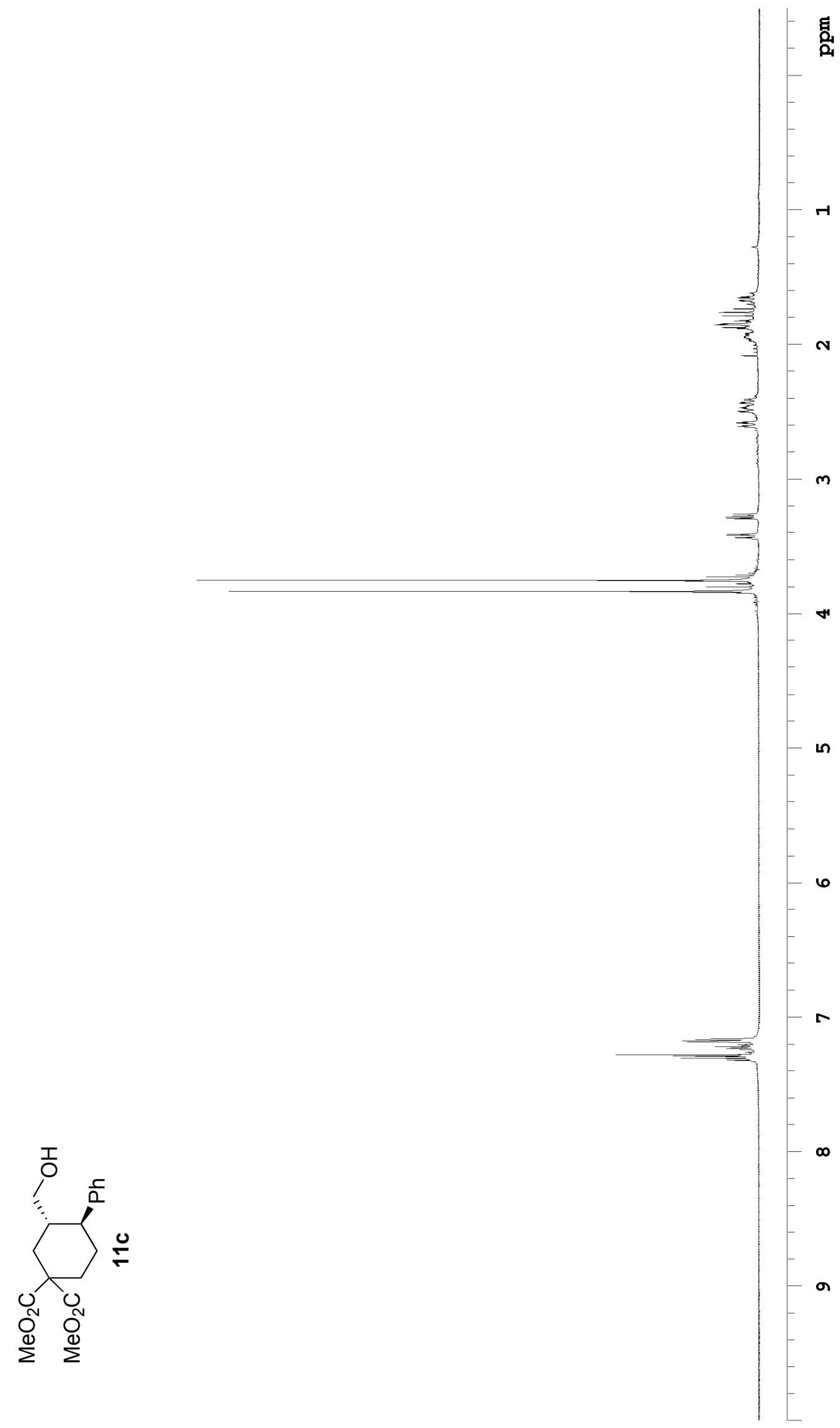




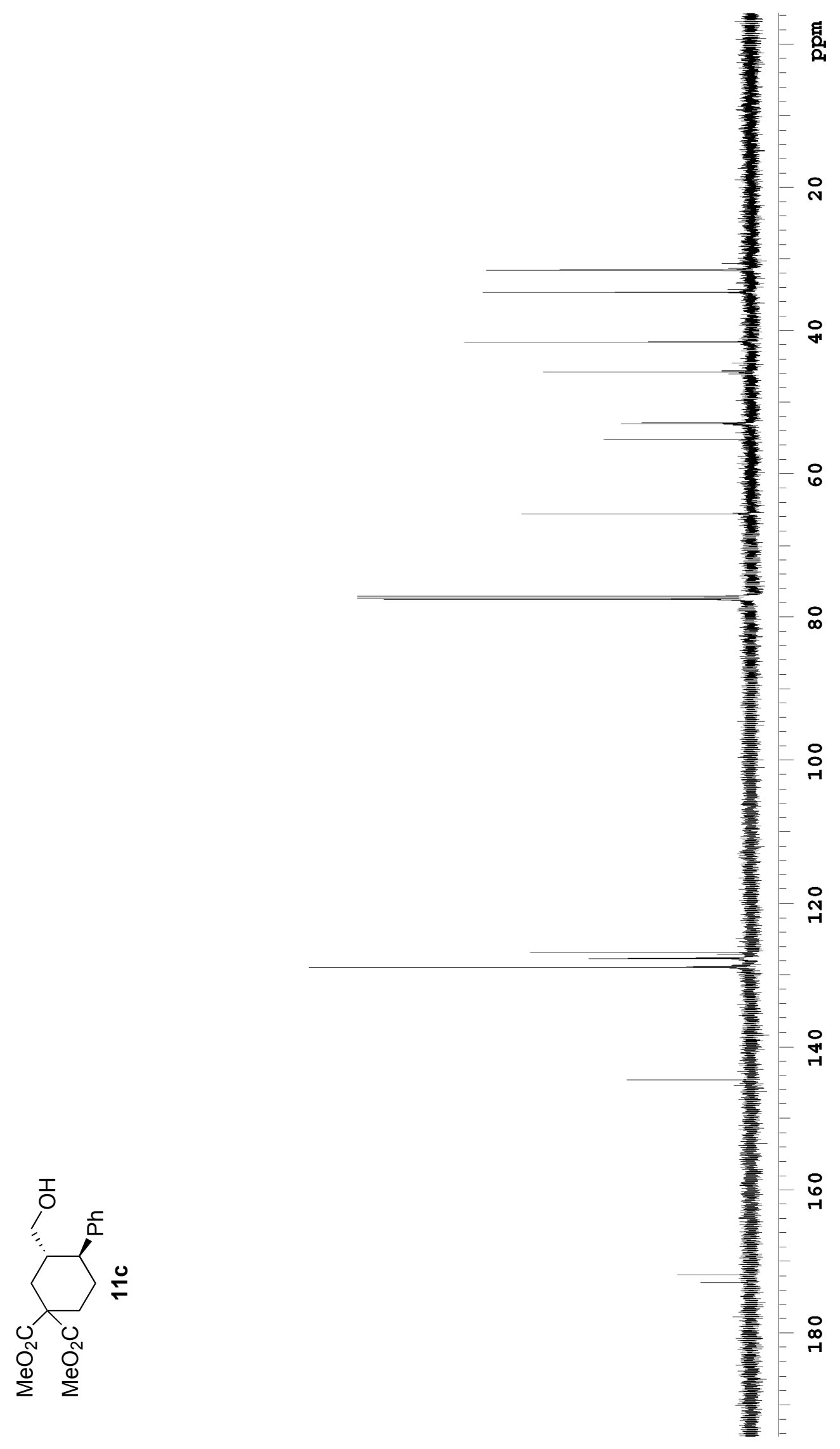



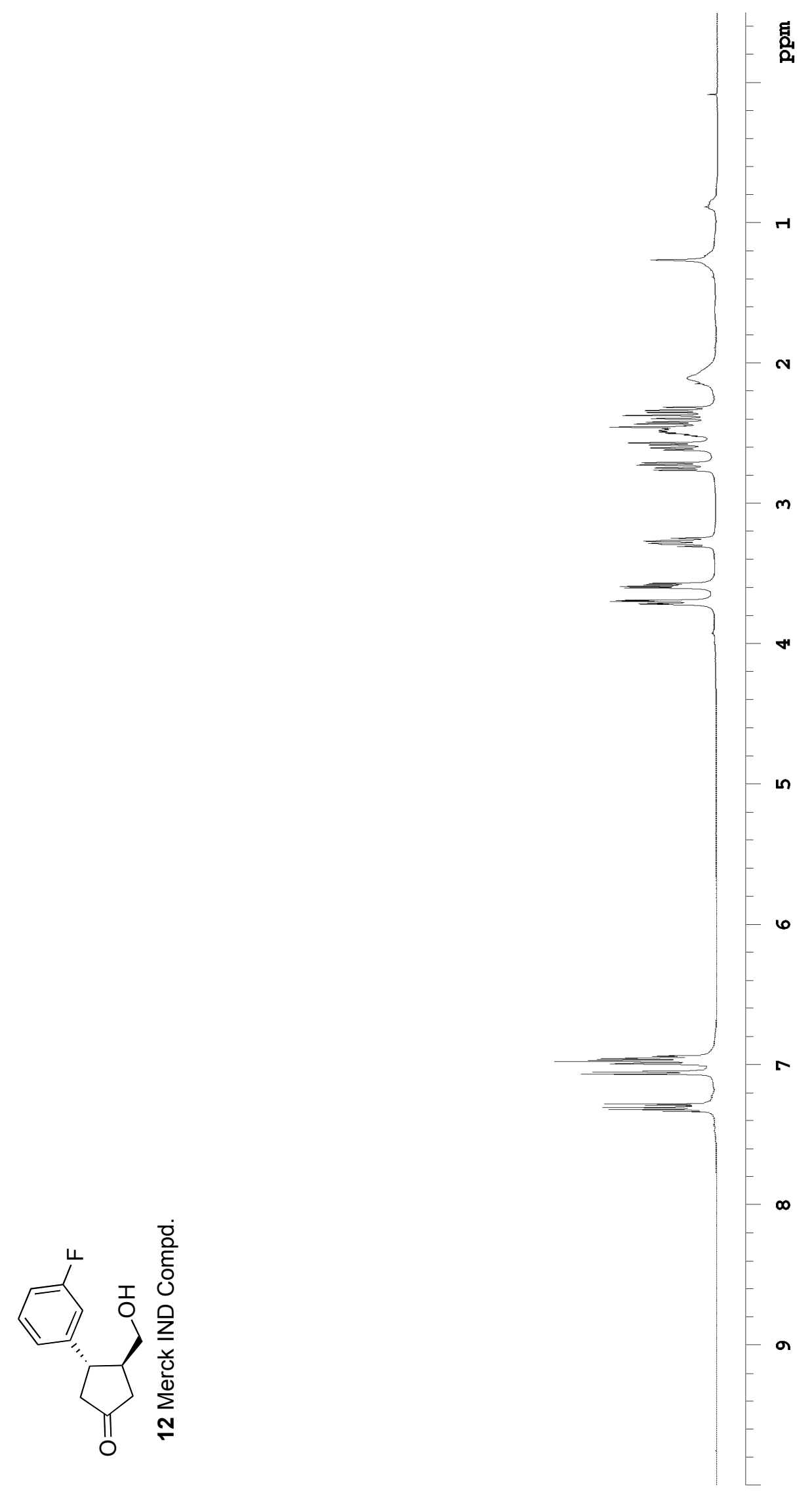

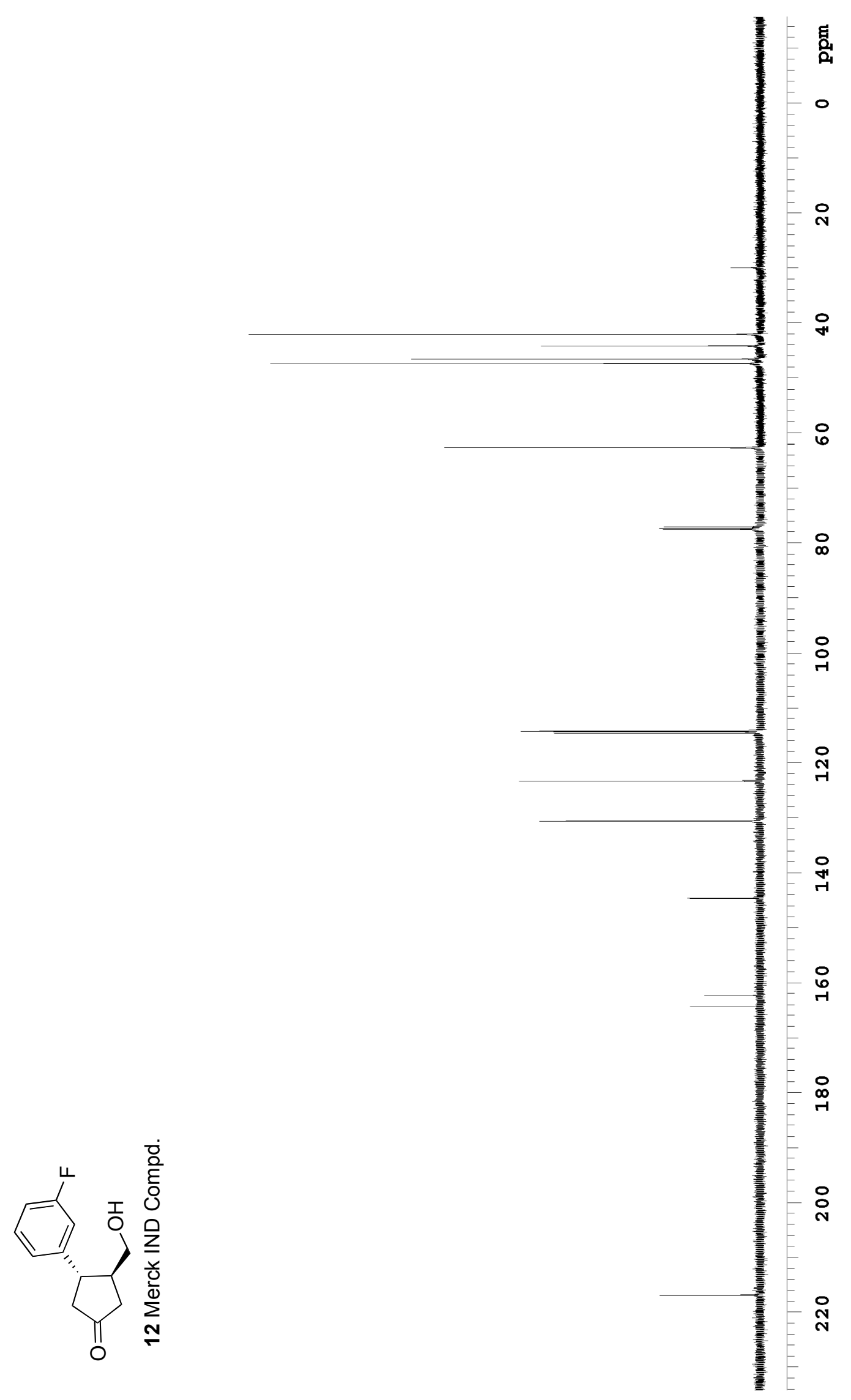


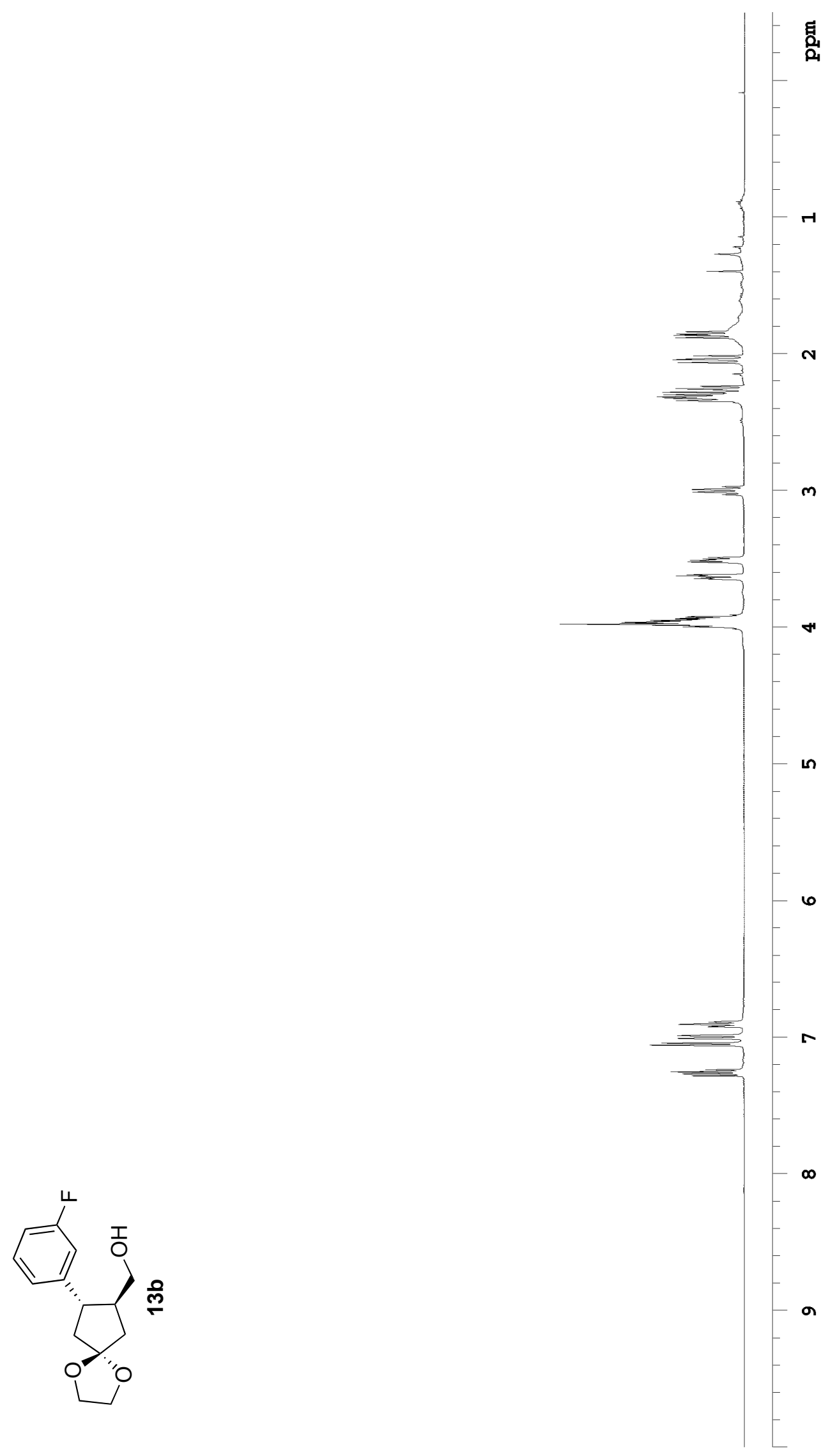




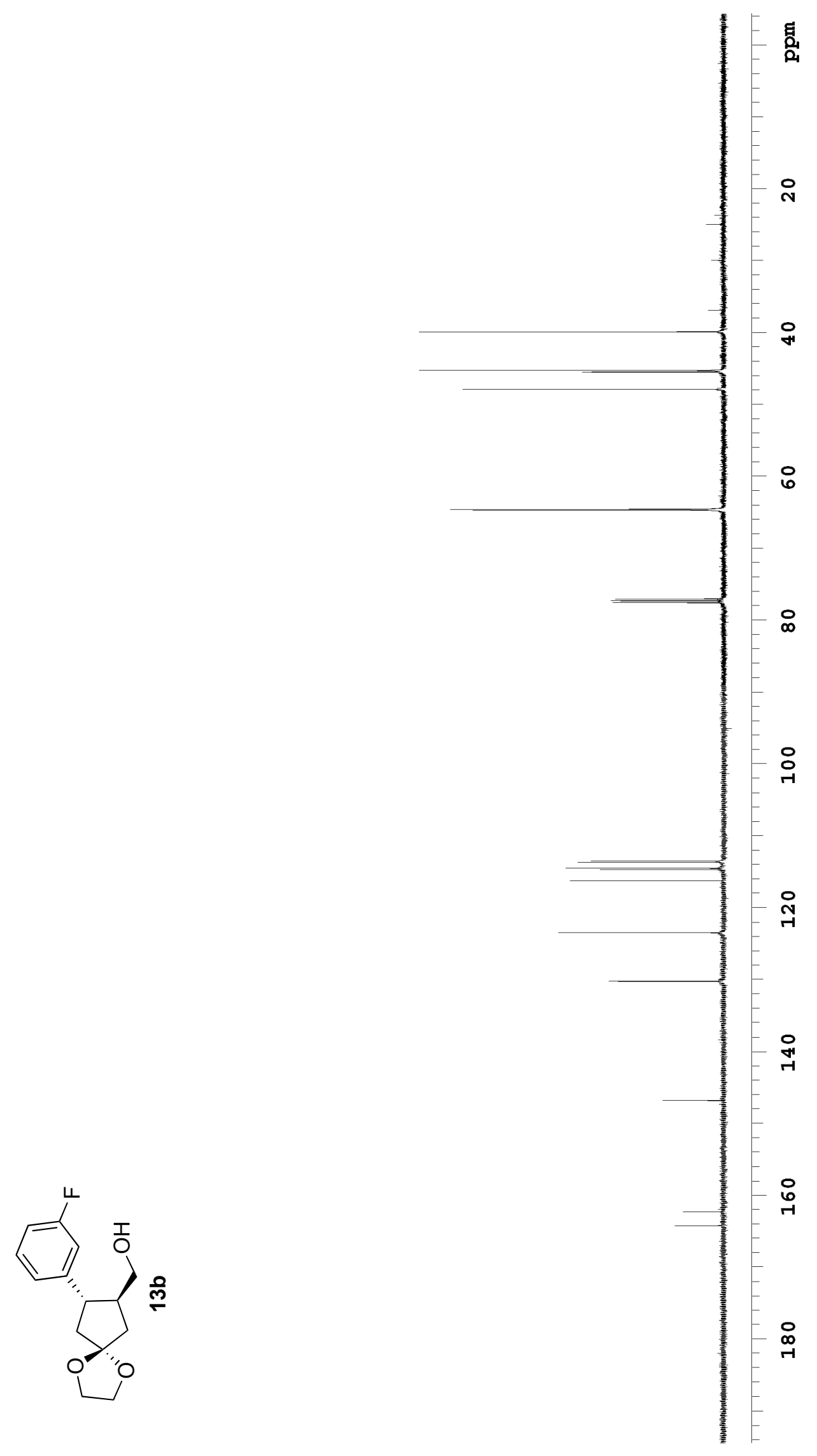




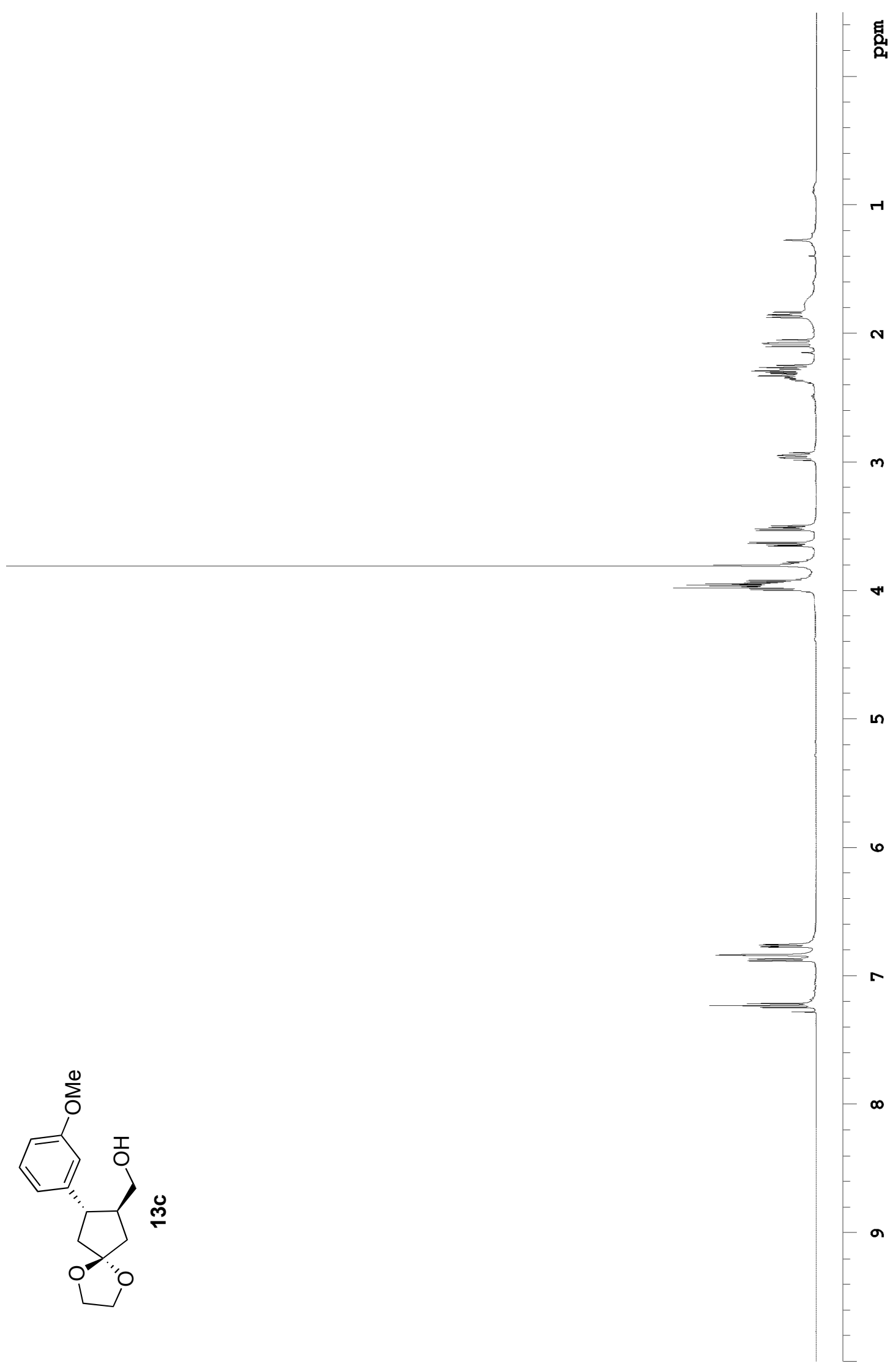




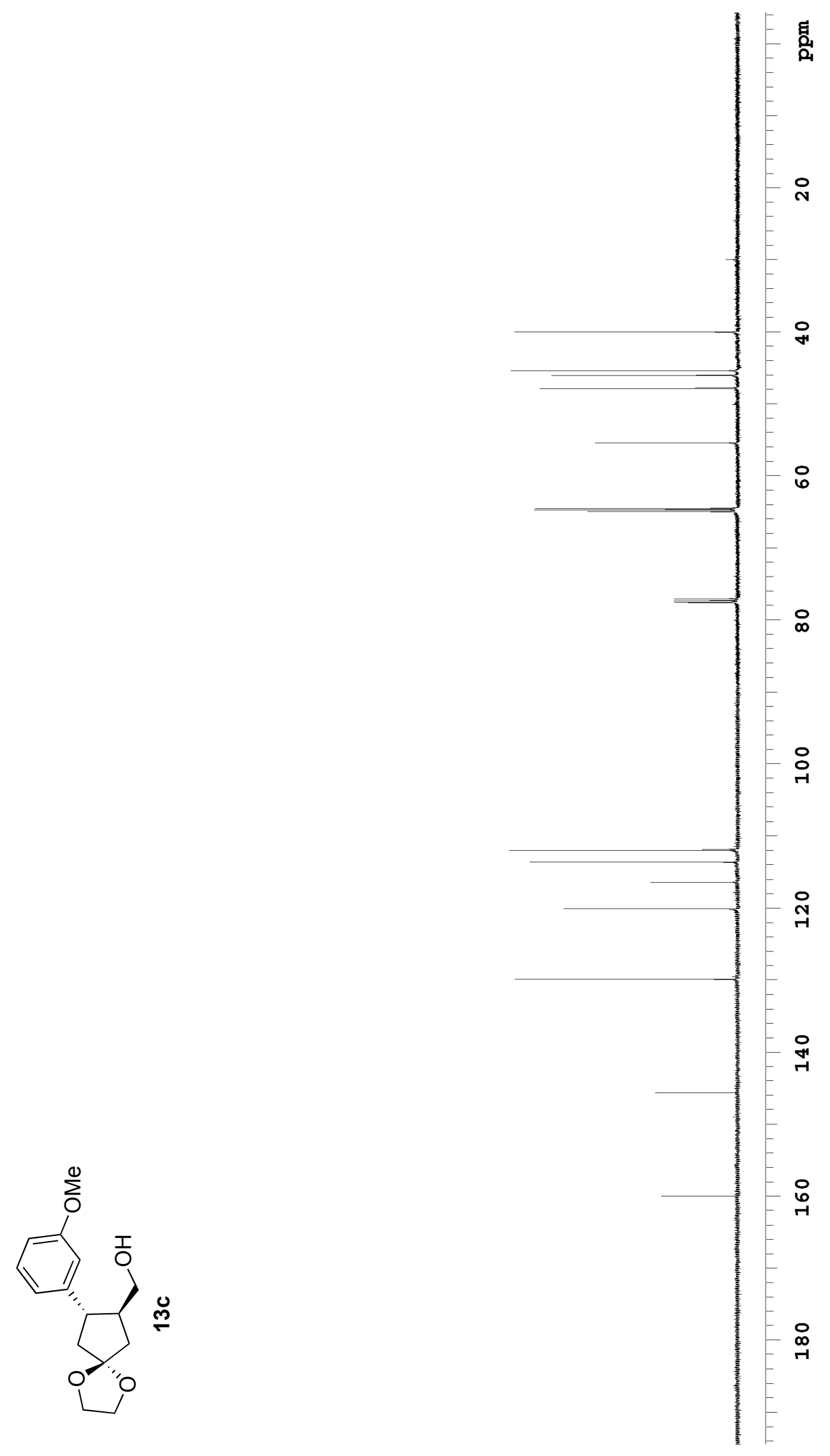

